ACCepted for publichtion in ApJ; March 20, 2021

Preprint typeset using $\mathrm{L}_{\mathrm{A}} \mathrm{X}$ style emulateapj v. 5/2/11

\title{
RESONANT CLUMPING AND SUBSTRUCTURE IN GALACTIC DISCS
}

\author{
Matthew Molloy \\ Kavli Institute for Astronomy \& Astrophysics, Peking University, \\ Yi He Yuan Lu 5, Hai Dian Qu, Beijing 100871, China \\ Martin C. Smith* And Juntai Shen* \\ Key Laboratory for Research in Galaxies and Cosmology, Shanghai Astronomical Observatory, \\ Chinese Academy of Sciences, 80 Nandan Road, Shanghai 200030, China \\ N. WYN Evans \\ Institute of Astronomy, Madingley Road, Cambridge, CB3 0HA, UK \\ Accepted for publication in ApJ; March 20, 2021
}

\begin{abstract}
We describe a method to extract resonant orbits from N-body simulations exploiting the fact that they close in frames rotating with a constant pattern speed. Our method is applied to the N-body simulation of the Milky Way by Shen et al. (2010). This simulation hosts a massive bar, which drives strong resonances and persistent angular momentum exchange. Resonant orbits are found throughout the disc, both close to the bar and out to the very edges of the disc. Using Fourier spectrograms, we demonstrate that the bar is driving kinematic substructure even in the very outer parts of the disc. We identify two major orbit families in the outskirts of the disc, one of which makes significant contributions to the kinematic landscape, namely the $m: l=3:-2$ family resonating with the bar.

A mechanism is described that produces bimodal distributions of Galactocentric radial velocities at selected azimuths in the outer disc. It occurs as a result of the temporal coherence of particles on the $3:-2$ resonant orbits, which causes them to arrive simultaneously at pericentre or apocentre. This resonant clumping, due to the in-phase motion of the particles through their epicycle, leads to both inward and outward moving groups which belong to the same orbital family and consequently produce bimodal radial velocity distributions. This is a possible explanation of the bimodal velocity distributions observed towards the Galactic anti-Centre by Liu et al. (2012). Another consequence is that transient overdensities appear and dissipate (in a symmetric fashion) resulting in a periodic pulsing of the disc's surface density.

Subject headings: Galaxy: kinematics and dynamics - Galaxy: disk - Galaxy: bulge - galaxies: kinematics and dynamics - galaxies: evolution - galaxies: bulges
\end{abstract}

\section{INTRODUCTION}

Modelling the stellar disc in spiral galaxies often begins with the assumption of axisymmetry. For some purposes, this can be sufficient and is often illuminating and powerful. Real spiral galaxies however are not so simple and exhibit a wealth of structure leading to strong non-axisymmetries. The Milky Way is host to numerous non-axisymmetric features such as spiral arms and the central bar. It has been shown that the majority of bright spirals are host to strong bars (e.g., Eskridge et al. 2000, Marinova \& Jogee 2007). N-body simulations of disc galaxies that start out in an axisymmetric configuration often succumb to dynamical instabilities and self-consistently develop bars and spirals (e.g., Sellwood 1981; Debattista et al. 2006; Martinez-Valpuesta et al. 2006). These features can have have a significant effect on the orbital structure in discs and so it is of great importance to understand these mechanisms and have the right tools at our disposal to analyse models that exhibit these features.

Orbits in non-axisymmetric systems periodically experience torques introduced by rotating overdensities. Long

\footnotetext{
^Corresponding Authors: matthewmolloy@gmail.com (MM), msmith@shao.ac.cn (MCS), jshen@shao.ac.cn (JS), nwe@ast.cam.ac.uk (NWE)
}

lived features allow time for orbits to become resonantly coupled with periodic variations in the torque as the disc evolves. Naturally, this means that the locations of these resonances are strongly linked to the pattern speed of the rotating features and the rotation curve of the disc. The important locations are the resonant radii such as at corotation (CR) and the location of the Lindblad resonances (LR) (see, for example, Weinberg 1994; Binney \& Tremaine 2008). At these locations, the orbits ditter drastically trom those in axisymmetric models. Deviations from axisymmetry, even in the disc centre, therefore cannot be disregarded when trying to explain kinematic structure in the outskirts of the disc. While it has been shown in external galaxies and in simulations that a central bar cannot extend beyond CR (Chirikov 1979. Contopoulos 1980, Sellwood \& Wilkinson 1993), it can have observable effects and influence on the dynamics even in the outer parts of the disc.

It is now beyond doubt that the Milky Way is host to a central bar (e.g., Blitz \& Spergel 1991; Dwek et al. 1995), yet its characteristic properties are still the subject of debate (e.g., Dehnen 1999, Gerhard \& Martinez-Valpuesta 2012). Resonant features induced by the bar have been used to explain the nature of moving groups in the Solar vicinity seen in the Hipparcos and Geneva-Copenhagen 
Survey data (Dehnen 2000, Minchev et al. 2010). Departures from axisymmetry in the disc have also been suggested as the reason for high values of the vertex deviation in the Solar neighbourhood and also low values for the ratio of the principal axes of the velocity-dispersion tensor (Evans \& Collett 1993. Dehnen \& Binney 1998). Larger scale surveys such as the RAVE (RAdial Velocity Experiment; Steinmetz et al. 2006) have recently been used to map the kinematic landscape in the Solar neighbourhood (e.g., Siebert et al. 2011; Monari et al. 2014), but the lack of accurate parallaxes and proper motions prohibit an investigation into full 6-D phase-space structures over significantly broad areas of the disc. The Sloan Digital Sky Survey (SDSS; Ahn et al.2012) and in particular the Sloan Extension for Galactic Understanding and Exploration (SEGUE; Yanny et al. 2009a) has been of immense value in describing the properties of the Milky Way disc (e.g., Carollo et al. 2010. Bond et al. 2010 Bovy et al. 2012a b; Smith et al. 2012), in uncovering substructure in the Milky Way's stellar halo (e.g., Smith et al. 2009, Yanny et al. 2009b; de Jong et al. 2010) and in discovering new Milky Way satellites and characterising their tidal tails (e.g., Newberg et al. 2009; Belokurov et al. 2010, Koposov et al. 2010). More recently the APUGEE (Apache Point Ubservatory Galactic Evolution Experiment; Eisenstein et al. 2011; Majewski \& SDSS-III/APOGEE Collaboration 2014) infrared (IR) survey has probed the low latitude regions of the disc, heavily obscured in optical surveys. This large scale IR survey of the Galactic disc (mostly bright giants and red clump (RC) stars) has allowed us to identify kinematic features associated with the bar (e.g., Nidever et al. 2012; Vásquez et al. 2013), but without accurate distances on the largest scales their interpretation is still the subject of debate (see $\mathrm{Li}$ et al. 2014). On smaller scales, but with more accurate distances, the RC sample (Bovy et al. 2014b) has yielded valuable insights into broad streaming motions present in the disc (Bovy et al. 2014a) as well as motions associated with the spiral arms (Kawata et al. 2014) and the Galactic warp (Lopez-Corredoira et al. 2014). Beyond positions and velocities, spectroscopic surveys also permit a view of the chemical landscape of the disc (Randich et al. 2013. Rojas-Arriagada et al. 2014; Howes et al. 2014; Nidever et al. 2014). Obtaining accurate $6 \mathrm{D}$ phase space intormation along with abundances allows us to probe the evolutionary history of the Milky Way.

Orbits in stellar discs are often described by oscillations in the radial, azimuthal and vertical directions with respective frequencies, $\kappa, \Omega$ and $\nu$. Non-axisymmetric patterns are also characterised by the frequency with which they rotate in the disc, often called the pattern speed, $\Omega_{p}$. A resonance occurs (in the plane of the disk) when there is a commensurability between these frequencies, namely

$$
l \kappa=m\left(\Omega-\Omega_{\mathrm{p}}\right)
$$

or equivalently,

$$
l T_{\phi}^{\prime}=m T_{R}
$$

where $l$ and $m$ are some integer values and $T_{R}$ and $T_{\phi}^{\prime}$ are the epicyclic and the orbital period in the rotating frame respectively. The component $\Omega-\Omega_{\mathrm{p}}$ is the orbital frequency of the star in the frame rotating with pattern speed $\Omega_{\mathrm{p}}$. In this frame, the orbit completes $m$ radial oscillations for every $l$ azimuthal oscillations. As $m$ and $l$ are integers, the resonant orbit closes in the rotating frame. The integer $m$ also describes the multiplicity of the pattern the closed orbit traces out - the orbit will have $m$-fold symmetry after $l$ rotations. We note here that, outside of CR, the orbit proceeds in an opposite sense to the pattern with $\Omega_{\mathrm{p}}$. For this reason, we set $l<0$ outside CR. With this terminology, the Outer LR (OLR) corresponds to the $m: l=2:-1$ resonance where $\Omega-\Omega_{\mathrm{p}}$ is necessarily negative, so by keeping $l<0$ in this region (beyond CR) Equation 1 is satisfied. At the OLR then, an orbit completes 2 radial oscillations for every one azimuthal oscillation when viewed in the rotating frame with angular frequency $\Omega_{\mathrm{p}}$.

The study of dynamics near resonances has a long history (e.g., Contopoulos 1970, Lynden-Bell \& Kalnajs 1972: Lynden-Bell 1973). 'The classical approach involves perturbations to an axisymmetric model (see Lichtenberg \& Lieberman 1983). This line of inquiry gives valuable insights into the dynamics of orbits under small perturbations and is often used in studying linear stability (e.g., Gerhard \& Saha 1991; Palmer 1994). The addition of a perturbation series however encounters a problem near resonances. It involves the addition of a component with a divisor equal to $l \kappa-m\left(\Omega-\Omega_{\mathrm{p}}\right)$, which of course approaches zero at a resonance - the well-known "problem of small divisors" (see e.g., Arnol'd 1963). This results in a divergence of the solution near a resonance and so does not give a formal answer.

The analysis of resonances in N-body simulations is usually handled by means of a frequency analysis. This requires either measuring the instantaneous frequencies of particles in the simulation or integrating the chosen orbit in a frozen potential that corresponds to the density distribution at some timestep in the simulation. Since the emergence of large N-body simulations, this method has become ubiquitous and has proved to be powerful and enlightening. However, the method assumes that angular momentum exchange is minimal and has no significant effect over the course of the orbit. This may lead to errors if angular momentum exchange is both constant and significant. In a potential with a significant perturber, instantaneous frequencies do not give give an accurate representation of the full orbit. This is especially true in the case of resonant orbits in discs with large bars where angular momentum is constantly, and periodically, lost and gained. For this reason, the azimuthal frequency must be calculated over the whole orbit, preferably over one rotation in the rotating frame. Below we outline a simple method to get a robust estimate of $\Omega$ (Section 3.1) using only the simulation output.

A Fourier analysis is often employed to extract frequencies and is usually sufficient, but in some cases encounters a problem in the outskirts of the disc where frequencies are low. A slowly changing potential can introduce low frequency radial modes and so a lower cutoff is introduced to remove these, but may also remove some orbits in the very outskirts of the disc (Ceverino \& Klypin 2007). As we shall see below (Section 4.1), the potentials of N-body simulations rarely have the characteristic of being time independent. This means that integrating an orbit in a frozen potential will fail to replicate the real N-body trajectory. Also, it is well known that angular momentum exchange between a galactic bar and the halo or a gaseous component to the disc can vary the pattern speed and strength of the bar (Athanassoula 2002,2003). By choosing a time-independent pattern speed, orbits integrated in frozen potentials loose this characteristic of realistic barred potentials. 


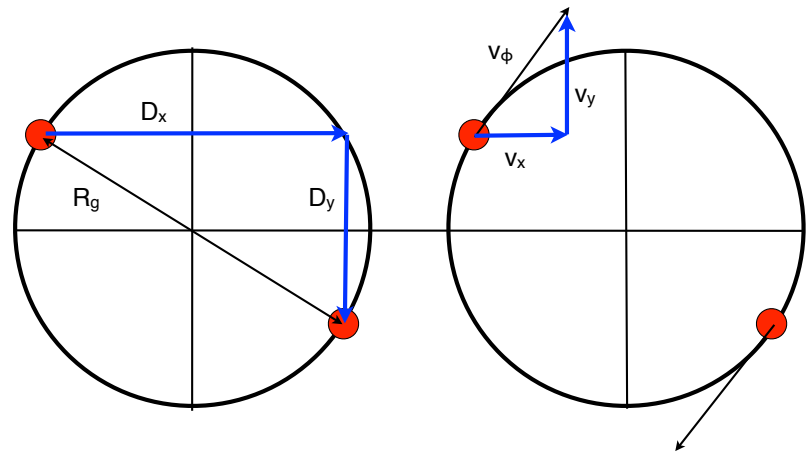

Figure 1. Circular orbits suggests a normalisation for our phase space distance metric. The maximum separation on the $(x, y)$ plane between two circular orbits differing only in orbital phase is twice the guiding radius (left). Using this as our normalisation means the spatial component of the phase space distance varies between 0 and 1 . In a similar fashion, the maximum separation of the kinematic component is twice the rotational velocity (right). This normalisation forces the kinematic component of the phase space to vary between 0 and 1 also.

Below we introduce a new method for analysing Nbody simulations. Its main aim is to uncover resonant orbits in stellar discs using the fact that, in a rotating frame, the orbit should close and return to a previously occupied region of phase space. We outline the method in Section 2. The method requires little a priori information. It only requires the re-calculation of the N-body orbits in many different frames. This approach is then applied to the outskirts of the N-body simulation described by Shen et al. (2010) in Section 3. As a diagnostic tool, we will construct Fourier spectrograms for the disc (Section 3.2) and estimate the radial and azimuthal frequencies (Section 3.1). This is not required to extract a sample of resonant orbits from the simulation, but only aids in their interpretation. We then explore some of the properties of our extracted sample of resonant orbits in Section 4 and discuss our findings in Section 5

\section{PHASE-SPACE DISTANCE METRIC}

In a particular rotating frame, a resonant or periodic orbit forms a closed pattern and returns to a previously occupied patch of phase space. Using this, we make a blind search for orbits that return to some arbitrarily selected starting position in phase space. To do this, we re-calculate the orbits from our N-body simulation in many different rotating frames. We define a phase space distance metric, which measures the distance traveled in the rotating frame $D_{\mathrm{ps}}$. A resonant orbit closes in the rotating frame of its perturber and so $D_{\mathrm{ps}}$ for this orbit approaches zero. In practice, $D_{\mathrm{ps}}$ will not return exactly to zero so we define some cutoff point, below which the orbit has closed, or nearly so.

N-body simulations give the full orbital history in the inertial frame $\left(R, \phi, z, v_{R}, v_{\phi}, v_{z}\right)$, which can be recalculated in a rotating frame $\left(R^{\prime}, \phi^{\prime}, z^{\prime}, v_{R}^{\prime}, v_{\phi}^{\prime}, v_{z}^{\prime}\right)$. We first define our (arbitrarily chosen) starting position $t_{0}$ and from there calculate the positions and velocities of particles along their orbits in many rotating frames using a grid of pattern speeds. Since the radial position and velocity are invariant in frames rotating about the $z$-axis, the calculation of the orbit in the rotating frame is trivial. We can write

$$
\begin{aligned}
R_{i}^{\prime} & =R_{i} \\
v_{R, i}^{\prime} & =v_{R, i} \\
\phi_{i}^{\prime} & =\phi_{i}-i \Omega_{\mathrm{p}} \triangle t \\
v_{\phi, i}^{\prime} & =v_{\phi, i}-R_{i} \Omega_{\mathrm{p}}
\end{aligned}
$$

where $\Omega_{\mathrm{p}}$ is the frequency of the rotating frame, $\Delta t$ is the duration of the timestep (from the N-body simulation, $\Delta t=0.96 \mathrm{Myr}$ ) and $i$ is the number of timesteps away from the starting point of the calculation. To make a meaningful normalisation of phase space distances, we convert the positions and velocities from polar to Cartesian coordinates $\left(\left[R^{\prime}, \phi^{\prime}, v_{R}^{\prime}, v_{\phi}^{\prime}\right] \rightarrow\left[x^{\prime}, y^{\prime}, v_{x}^{\prime}, v_{y}^{\prime}\right]\right)$. For each particle, the distance along each axis of phase space at timestep $t_{i}$ is given by

$$
\begin{aligned}
D_{x, i} & =\left|x^{\prime}\left(t_{0}\right)-x^{\prime}\left(t_{i}\right)\right| \\
D_{y, i} & =\left|y^{\prime}\left(t_{0}\right)-y^{\prime}\left(t_{i}\right)\right| \\
D_{v_{x}, i} & =\left|v_{x}^{\prime}\left(t_{0}\right)-v_{x}^{\prime}\left(t_{i}\right)\right| \\
D_{v_{y}, i} & =\left|v_{y}^{\prime}\left(t_{0}\right)-v_{y}^{\prime}\left(t_{i}\right)\right| .
\end{aligned}
$$

Formally, phase space is not a metric space and so any distance measure is arbitrary and tailored to suit the problem under study. Our general rule of thumb is to first define the expected limits of the orbit in each dimension and normalise accordingly. In this case, we use expectations from simple circular disc orbits. We note that for almost circular orbits

$$
0 \lesssim \sqrt{D_{x}^{2}+D_{y}^{2}} \lesssim 2 R_{\mathrm{g}}
$$

where $R_{\mathrm{g}}$ is the guiding radius of the orbit (i.e. the radius of a circular orbit with an equivalent angular momentum). This suggests the following normalisation on the spatial component of the phase space,

$$
D_{\mathrm{p}, i}=\frac{\sqrt{D_{x, i}^{2}+D_{y, i}^{2}}}{2 \bar{R}_{\mathrm{g}}} .
$$

Since angular momentum exchange is ongoing in the disc, we use the average guiding radius over the course of the orbit, $\bar{R}_{\mathrm{g}}$ (note that the guiding radius is evaluated by equating the angular momentum, $L_{z}$, of the particle with the angular momentum of a rotation curve derived from azimuthally-averaged force calculations). However, the median guiding radius may serve as a better normalisation if sampling is far from uniform along the orbit. If an orbit near the centre of the disc is sampled with a constant $\triangle t$, then there will be significantly more points around apocentre than pericentre, skewing the value of $\bar{R}_{\mathrm{g}}$ to higher values. By using either the average or median $R_{\mathrm{g}}$, the spatial component of $D_{\mathrm{ps}}$ varies between roughly 0 and 1 (see Figure 1 ). We use a similar method to normalise the kinematic component of the phase space distance. For almost circular orbits, it is true that

$$
0 \lesssim \sqrt{D_{v_{x}}^{2}+D_{v_{y}}^{2}} \lesssim 2 v_{\phi}^{\prime}
$$

so we can calculate a locally normalised distance using

$$
D_{v, i}=\frac{\sqrt{D_{v_{x}, i}^{2}+D_{v_{y}, i}^{2}}}{2 \bar{v}_{\phi}^{\prime}} .
$$




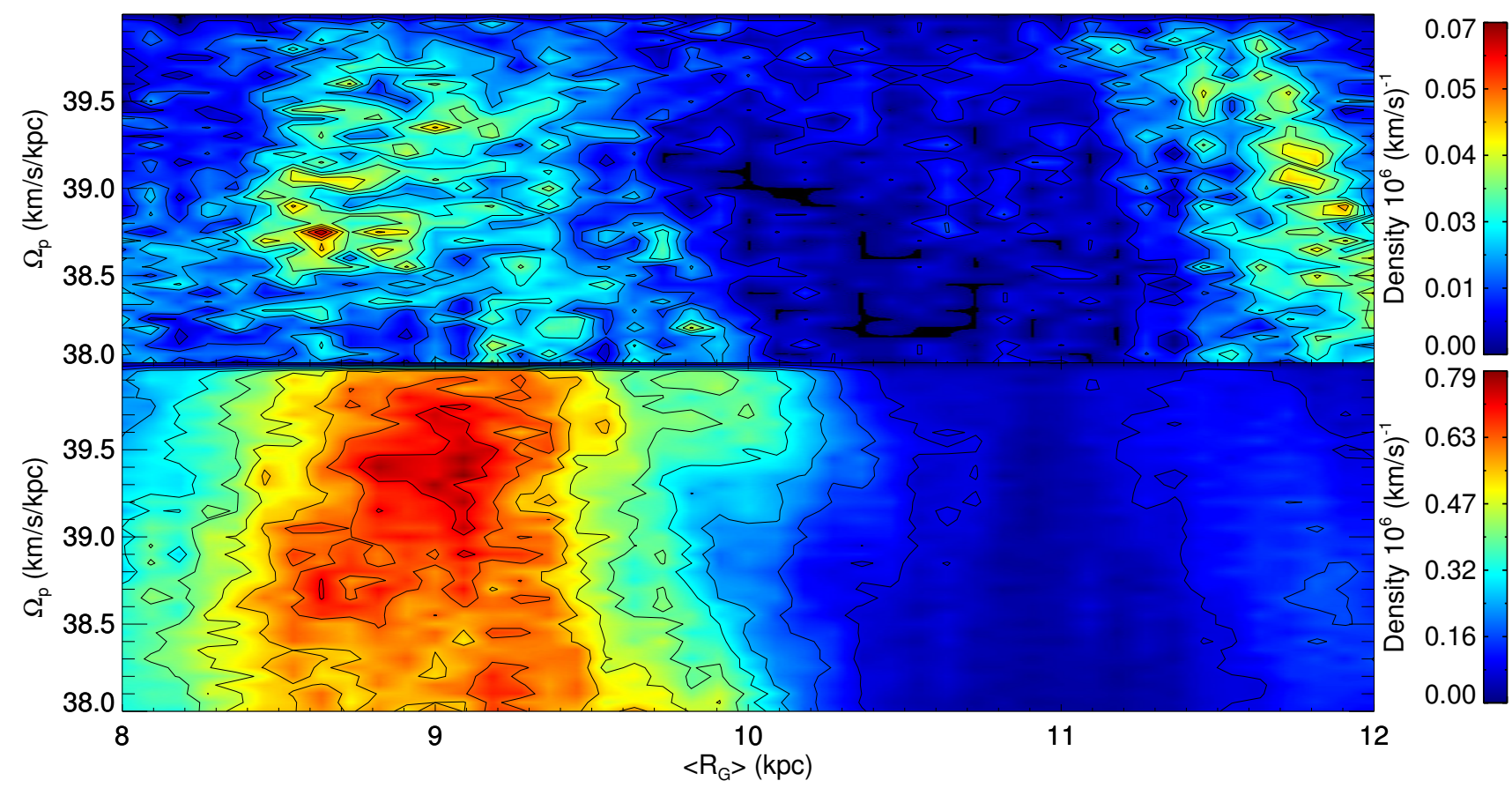

Figure 2. Density of closed orbits as a function of their average guiding radius and the pattern speed for which they close. We study various rotating frames with frequencies in the range $38<\Omega_{\mathrm{p}}<40 \mathrm{~km} \mathrm{~s}^{-1} \mathrm{kpc}^{-1}$ using a grid of $\triangle \Omega_{\mathrm{p}}=0.1 \mathrm{~km} \mathrm{~s}^{-1} \mathrm{kpc}^{-1}$. The top panel indicates our selection when the minimum phase space achieved is less than $D_{\mathrm{ps}}<0.025$, and the bottom when $D_{\mathrm{ps}}<0.04$. Most of the closed orbits have $8<R_{\mathrm{g}}<10 \mathrm{kpc}$ with a smaller contribution from orbits with $R_{\mathrm{g}}>11 k p c$.

\begin{tabular}{cccc}
\hline \hline Cut $\left(D_{\mathrm{ps}}<\mathrm{Cut}\right)$ & Total & $R \leq 5 \mathrm{kpc}$ & $R>5 \mathrm{kpc}$ \\
\hline No Cut & 977,357 & 680,005 & 297,352 \\
0.1 & 529,015 & 424,097 & 104,918 \\
0.08 & 425,788 & 355,854 & 69,934 \\
0.06 & 297,186 & 255,247 & 41,939 \\
0.04 & 141,061 & 121,747 & 19,314 \\
0.025 & 40,744 & 35,478 & 5,266
\end{tabular}

Table 1

By imposing stricter cuts on the minimum phase space distance $D_{\text {ps }}$, we extract samples of cleaner, more strictly closed, orbits.

Here, we have again taken an average of $v_{\phi}$ in the rotating frame over the course of the orbit (a median value may be more appropriate in the inner parts of the disc). As with the spatial component, using $\bar{v}_{\phi}^{\prime}$ means that the kinematic component of $D_{\mathrm{ps}}$ varies between roughly 0 and 1 (see Figure 1). The full phase space distance is then calculated as

$$
D_{\mathrm{ps}, i}=\sqrt{D_{\mathrm{p}, i}^{2}+D_{\mathrm{v}, i}^{2}}
$$

where $D_{\mathrm{ps}}$ varies roughly between 0 and $\sqrt{2}$.

This normalisation does introduce separate biases on the spatial and kinematic components. On the spatial plane, the normalisation is biased in favour of particles that gain angular momentum over the course of the orbit. A significant gain in angular momentum gives rise to an increase in the guiding radius, thereby lowering the spatial phase space distance $\left(D_{p}\right)$ measured. In a similar vein, an increase in $v_{\phi}^{\prime}$ gives rise to a lower kinematic phase space distance. We have checked that this bias is weak for reasonable values of $D_{\mathrm{p}}$ and only becomes important when $D_{\mathrm{p}}$ is a significant fraction of the guiding radius $\left(D_{\mathrm{p}} \geq R_{\mathrm{g}} / 2\right)$ - at this point the orbit will have been excluded by any cut made on $D_{\mathrm{ps}}$. In any case, resonant orbits experience periodic changes in angular momentum as they complete their orbits, so for closed orbits, the variations cancel.

By measuring the lowest phase-space distance of each particle, we can garner a sample of closed orbits by making a cut below which the orbits are deemed to have closed. In order to diminish the effects of using an arbitrary starting point, which can give spurious results, we use three different starting points $\left(t_{0}\right)$. We only accept orbits that have a minimum phase-space distance below our predefined cut using all three starting points. Each starting point is separated by 200 timesteps or $\approx 200$ Myr, which constitutes a significant fraction of the orbital period. Using this separation means we choose very different orbital phases as our starting points, except of course for those with $T_{\phi}^{\prime} \approx 200 \mathrm{Myr}$.

\section{AN APPLICATION TO AN N-BODY SIMULATION}

We test this method using the N-body simulation described by Shen et al. (2010). This simulation begins as an axisymmetric disc which, due to dynamical instabilities (see Hohl 1971: Kalnajs 1978; Toomre 1981: Sellwood 1981), develops a strong bar that persists for $\sim 4.5$ Gyr. The pattern speed of the bar remains relatively stable at $\sim 38.5 \mathrm{~km} \mathrm{~s}^{-1} \mathrm{kpc}^{-1}$ for the duration of the simulation, since there is no gaseous disc component or live halo to which angular momentum can be deposited. The amplitude of the bar in this case is expected to result in a strong resonant reaction in the disc. The bar also drives recurring transient spirals. This persistent strong bar gives us the opportunity to test the above method in a situation where angular momentum exchange is significant and long lived, i.e., where a perturbation series and a frequency analysis may have difficulty. The corotation 
radius $(\mathrm{CR})$ of the bar is at $\approx 4.5 \mathrm{kpc}$ and the outer Lindblad resonance (OLR) lies in the range $8<R<9$ kpc.

The starting configuration of the simulation is an axisymmetric disc embedded in a logarithmic halo with a disc scalelength of $h_{R}=1.9 \mathrm{kpc}$ and scaleheight of $h_{z}=0.2 \mathrm{kpc}$. The disc is comprised of $10^{6}$ stellar particles with a total mass of $M_{\mathrm{D}}=4.25 \times 10^{10} M_{\odot}$, while the mass of the halo is determined by an asymptotic rotational velocity of $v_{M}=250 \mathrm{~km} \mathrm{~s}^{-1}$. The simulation was tailored to match the kinematics of the Galactic bulge as observed by BRAVA (Bulge RAdial Velocity Assay; Rich et al. 2007; Howard et al. 2008) and this was used to constrain the angle of the bar with respect to the Galactic centre - Solar position line. The final configuration of the system consisted of a bar with a half-length of 4 $\mathrm{kpc}$ and an axial ratio of $\approx 0.5$, which is consistent with previous estimates from gas dynamics (Englmaier \& Gerhard 1999: Weiner \& Sellwood 1999). The final bar angle was $\sim 20^{\circ}$ again in agreement with previous studies (e.g., Stanek et al. 1997; Bissantz \& Gerhard 2002). It has also been recently shown that the simulation is in good agreement with observations of Red Clump (RC) stars towards the Galactic centre. An X-shaped structure that results from the buckling of the bar (see e.g. Raha et al. 1991) leads to a bimodal distribution of distances (or magnitudes) for some fields towards the Galactic centre (Saito et al. 2011) and is well replicated by both this simulation (Li \& Shen 2012) and that of Gardner et al. (2014). In a forthcoming paper (Molloy et al. in prep), we isolate the resonant orbits that contribute to this feature, describing their kinematic properties.

We apply the method over many epochs during the lifetime of the simulation, finding a good sample of closed orbits at all times after $\sim 2$ Gyr. We focus here on the last Gyr of the simulation ( $\sim 4-5$ Gyr). As we are particularly interested in orbits resonant with the bar, we limit out search to pattern speeds close to that of the bar $\left(38 \leq \Omega_{\mathrm{p}} \leq 40 \mathrm{~km} \mathrm{~s}^{-1} \mathrm{kpc}^{-1}\right)$. In Figure 2 we plot the density of closed orbits as a function of their average guiding radii and the pattern speed of the rotating frames in which they close. In the upper panel, we impose a cut of $D_{\mathrm{ps}}<0.025$ and in the lower, a cut of $D_{\mathrm{ps}}<0.04$. By imposing a tighter cut on $D_{\mathrm{ps}}$, we extract a "cleaner" sample of resonant orbits. As the cut approaches zero, we converge on the "parent" orbit for each resonance. Looser cuts then encompass orbits that librate about the parent orbit. In Table 1, we list the number of resonant orbits extracted for each cut. There are many more resonant orbits within CR. The choice of cut therefore depends on the region in question.

Here, we extract a sample of closed orbits whose distribution of guiding radii peaks at $\sim 9.0 \mathrm{kpc}$ and close in a rotating frame close to the pattern speed of the bar $\left(\sim 38.5 \mathrm{~km} \mathrm{~s}^{-1} \mathrm{kpc}^{-1}\right)$. In order to measure the pattern speed, we compute the numerical derivative of the phase angle of the $m=2$ Fourier component within CR over a number of timesteps. The pattern speed of the bar remains constant at $\approx 38.5 \mathrm{~km} \mathrm{~s}^{-1} \mathrm{kpc}^{-1}$ for most of the duration of the simulation (see also Section 3.2).

A source of ambiguity remains, however. An orbit can close in many frames. Specifically, an orbit with epicyclic $(\kappa)$ and orbital frequency $(\Omega)$ closes in a frame $\Omega_{m: l}$ for which

$$
\Omega_{m: l}=\Omega-\left(\frac{l}{m}\right) \kappa
$$

Here, the subscript $m: l$ indicates that the orbit closes with $m$ radial oscillations for every $l$ azimuthal oscillations in that frame. When we reproduce Figure 2 over a much wider range of pattern speeds $\left(25 \leq \Omega_{\mathrm{p}} \leq 55 \mathrm{~km}\right.$ $\mathrm{s}^{-1} \mathrm{kpc}^{-1}$ ), we see multiple overdensities corresponding to this degeneracy. So, while we have limited our search for resonant orbits associated with the bar, we may also be extracting orbits that are driven by slower or faster moving patterns and which also happen to close in a frame close to that of the bar's. To clarify the matter, we measure the orbital and epicyclic frequencies for our sample of closed orbits. Using this information, we determine the pattern speeds of the rotating frames in which our orbits will close for various families of orbits (e.g., $m: l=1:-1,1:-2$, etc.).

\subsection{Measuring Frequencies}

It is difficult to estimate reliably the orbital frequencies of stars in discs in which angular momentum exchange is persistent and significant. Taking instantaneous measurements of the azimuthal frequency $\Omega$ introduces an error since the angular momentum at one point in the orbit may be, sometimes significantly, different from that at another point. For this reason, it is better to estimate the frequency over the course of a full orbit. This is especially true for resonant orbits - the periodic loss and gain of angular momentum is averaged out over some integer number of azimuthal oscillations in the rotating frame.

Our method makes use of the fact that an orbit plotted in a rotating frame with the same frequency as the orbital frequency of its guiding centre will trace out an ellipse (its epicycle) which appears stationary. In this frame, the epicycle doesn't precess around the centre of the disc and explores only a limited range in $\phi$. In each rotating frame, the orbit has a minimum and maximum azimuth $\left(\phi_{\min }\right.$ and $\left.\phi_{\max }\right)$. The range in $\phi$ traversed by the orbit is $\Delta \phi=\left|\phi_{\max }-\phi_{\min }\right|$. The method minimises $\Delta \phi$ by scanning various rotating frames with higher and higher resolution until the variation between adjacent frames reaches some predefined limit. For well-behaved orbits, this converges to the true orbital frequency of the guiding radius. The epicyclic frequency is extracted from the period of the radial oscillation.

\subsection{Fourier Components}

Discerning which of the non-axisymmetric features of the disc (e.g., the central bar or spiral arms) resonate with our closed orbits requires an analysis of the wave patterns that propagate through the disc. For this, we decompose the disc into its Fourier components. We follow the procedure outlined by Quillen et al. (2011) to construct our spectrograms. For equally spaced radial bins at each timestep, we measure

$$
\begin{aligned}
W_{\mathrm{C}}(r, t) & =\sum_{j} \cos \left(\phi_{j}\right) \\
W_{\mathrm{S}}(r, t) & =\sum_{j} \sin \left(\phi_{j}\right)
\end{aligned}
$$

where we sum over all $j$ particles in each radial bin. We then numerically integrate over the complex function

$$
\tilde{W}(\omega, t)=\int_{T_{1}}^{T_{2}} \exp (-i \omega t) h(t)\left[W_{\mathrm{C}}(r, t)+i W_{\mathrm{S}}(r, t)\right] d t
$$




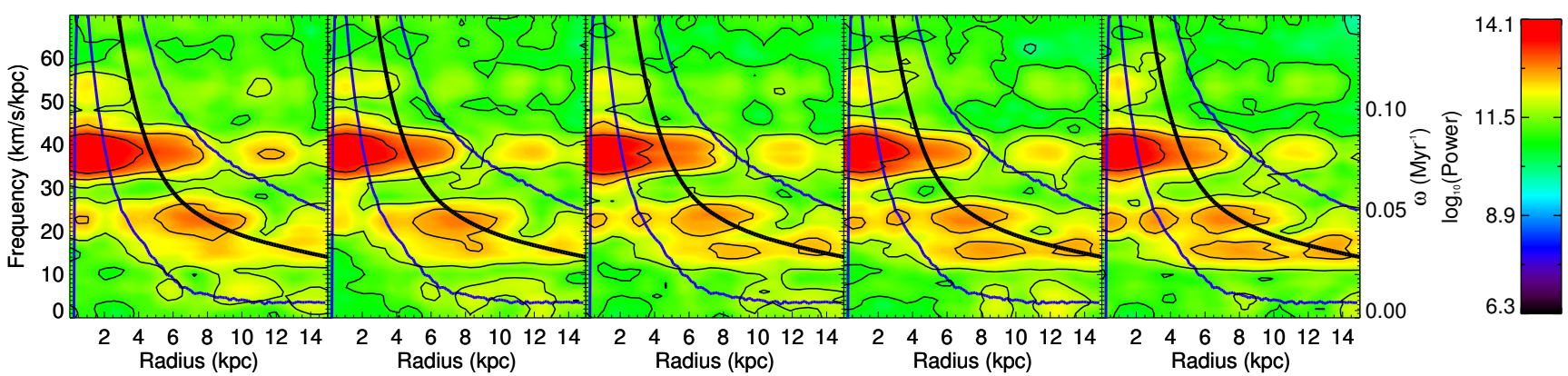

Figure 3. $m=2$ Fourier spectrograms spanning $T_{2}-T_{1}=1$ Gyr. From left to right, the spectrograms probe the time range from $2 \rightarrow$ 3 Gyr, $2.5 \rightarrow 3.5$ Gyr, $3 \rightarrow 4 \mathrm{Gyr}, 3.5 \rightarrow 4.5$ Gyr and $4 \rightarrow 5$ Gyr. In constructing our spectra, we sample the disc every $\sim 10 \mathrm{Myr}$ and use a Hanning window function to bracket $T_{1}$ to $T_{2}$ (Equation 12). The colourbar denotes a log scale in arbitrary units. We also indicate $\mathrm{CR}$ with the black line and the $2: \pm 1 \mathrm{LR}$ with the blue lines (Equations 13 and 14. The most prominent pattern is that of the bar which is rotating with a pattern speed of $\sim 38.5 \mathrm{~km} \mathrm{~s}^{-1} \mathrm{kpc}^{-1}$. It extends from the centre of the disc out to its CR at $\sim 4.5 \mathrm{kpc}$. A two-armed spiral emanates from the ends of the bar which also overlaps with a slower spiral feature at $\sim 25 \mathrm{~km} \mathrm{~s}^{-1} \mathrm{kpc}^{-1}$. We also see the emergence of a transient feature at $\sim 15 \mathrm{~km} \mathrm{~s}^{-1} \mathrm{kpc}^{-1}$.

where we sample over the frequency domain $0<\omega<70$ $\mathrm{km} \mathrm{s}^{-1} \mathrm{kpc}^{-1}$. We also use a Hanning window function $h(t)$ that spans the time window $T_{1}$ to $T_{2}$. We construct spectrograms using the $m=1,2,3$ and 4 Fourier components for five different time windows with $T_{2}-T_{1}=0.96$ Gyr (1,000 timesteps) from $\sim 2$ to $\sim 5$ Gyr (i.e. 2 Gyr $\rightarrow$ 3 Gyr, 2.5 Gyr $\rightarrow$ 3.5 Gyr, 3 Gyr $\rightarrow 4$ Gyr, etc.). The spectrograms are sampled every 9.6 Myr (10 timesteps) so that we can uncover transient features (such as spiral arms) that require a high temporal resolution.

The $m=1 \& 3$ spectrograms exhibit patterns many orders of magnitude weaker than the $m=2$ patterns, whilst the $m=4$ spectrograms show only the first harmonic of the $m=2$ patterns. We give only the $m=2$ spectrograms in Figure 3 with the vertical axis indicating the pattern speed and the horizontal axis the radial extent of the features. The colour shows the strength of the feature (in arbitrary units and on a log scale). We show $\mathrm{CR}$ as the black line and also the $2: \pm 1 \mathrm{LR}$ as blue lines. The $m: l$ LR are derived from the rotation curve calculated using force measurements around the disc. This gives $\Omega_{\mathrm{c}}(R)$, the azimuthal frequency of circular orbits at $R$. Using a numerical differentiation of $\Omega_{\mathrm{c}}^{2}$, we derive $\kappa_{\mathrm{c}}$ from

$$
\kappa_{\mathrm{c}}^{2}(R)=R \frac{d \Omega_{\mathrm{c}}^{2}}{d R}+4 \Omega_{\mathrm{c}}^{2}
$$

The $m: l$ Lindblad resonance (LR) is then (where, as before, $l<0$ for $R$ exceeding the corotation radius)

$$
L R_{m: l}(R)=\Omega_{\mathrm{c}}-\frac{l}{m} \kappa_{\mathrm{c}} .
$$

The spectrograms of the $m=2$ Fourier components indicate the radial extent and the rotational frequency of two-armed structures, like a bar or spiral arms, in the disc. The most prominent feature is the bar pattern, which extends from the centre of the disc out to its corotation radius, rotating at $\sim 40 \mathrm{~km} \mathrm{~s}^{-1} \mathrm{kpc}^{-1}$. A weaker feature extends from the end of the bar in all $m=2$ spectrograms and reaches as far as the 2:-1 LR. The orbits that make up the structure of the bar are prohibited from extending beyond corotation (see Chirikov 1979, Contopoulos 1980), so this feature is likely due to sp1rals emanating trom the ends of the bar. We see another two-armed structure that also extends from the end of the bar, but rotates with a lower frequency $\left(\sim 22 \mathrm{~km} \mathrm{~s}^{-1}\right.$ $\left.\mathrm{kpc}^{-1}\right)$. At later times, a further spiral is seen at a still lower frequency $\left(\sim 15 \mathrm{~km} \mathrm{~s}^{-1} \mathrm{kpc}^{-1}\right)$. This spiral has its ILR at the same radius as the bar's CR which may indicate the presence of mode-coupling (Tagger et al. 1987 Minchev et al. 2012). The bar is extremely stable in this simulation and only slows down very slightly over the last 4 Gyr. The spectrograms indicate clearly the presence of multiple patterns in the disc, which contribute to the ambiguity about the forcing resonant perturber.

\subsection{Diagnostic}

We now calculate the pattern speeds in which orbits close for various values of $m$ and $l\left(\Omega_{m: l}\right.$, Equation 10$)$. Using this along with our Fourier spectrograms, we can decipher the most likely resonant origin for our sample of closed orbits. We make two assumptions in the following analysis:

1. Resonant orbits occur due to non-axisymmetric patterns inside the guiding radii of the orbits.

2. Resonant orbits occur roughly at the location of a Lindblad Resonance/Radius (LR) (see Binney \& Tremaine 2008, section 3.3.3).

By plotting our $\Omega_{m: l}$ distributions over our $m=2$ spectrograms, we look for the coincidence of the distributions with non-axisymmetric features in the disc, satisfying our assumptions above. We neglect the $m=1,3 \& 4$ spectrograms since the amplitude of those patterns are dwarfed by the $m=2$ components.

Figure 4 shows the $m=2$ spectrograms for the final Gyr of the simulation (the right-most plot in Figure 3). The spectrograms are identical in each plot. We have overlain the distributions of $\Omega_{m: l}$ for the 1:-1, 2:-1, 3:-1 \& 3:-2 (first, second, third \& fourth plots respectively) orbit families as the blue dots. The blue dots in each of the plots mark the same particles. We have chosen only the particles that have $D_{\mathrm{ps}}<0.025$, namely the "cleanest", or "most-closed", orbits. Their different position in each plot represents the different frames in which they close as different types of orbits. We identify two separate groups of closed orbits with significantly different distributions in $\Omega_{m: l}$ - one lies inside $11 \mathrm{kpc}$, while the other lies outside. For both groups, we have plotted the median frequency in each distribution (given by the horizontal line). 


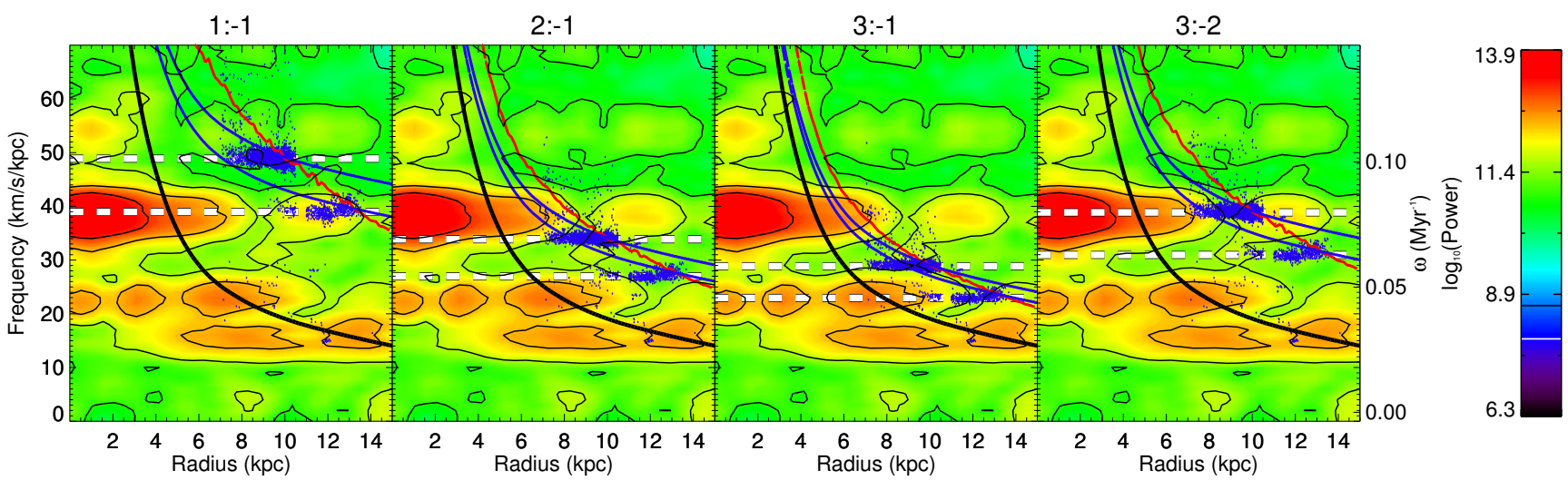

Figure 4. The $m=2$ component spectrogram covering the time window $4 \rightarrow 5$ Gyr, i.e. the right-most plot in Figure 3 Each plot shows the same spectrogram with the CR indicated as the black line. We indicate the $m: l$ LR as the blue and red lines - the 1:- 1 LR in the first plot, the 2:-1 LR in the second plot, and so on. The red line uses the epicyclic approximation (i.e. $\kappa$ is derived from the rotation curve, $\Omega_{\mathrm{c}}$ ), while the blue lines use the median $\kappa$ from samples of orbits. Specifically, the outer blue line uses the median $\kappa$ from the particles in the range $6<R<11 \mathrm{kpc}$, while the inner blue line uses the median $\kappa$ from particles with $R>11 \mathrm{kpc}$. In each plot, we overlay the distributions of $\Omega_{m: l}$ (Equation 10 for particles with $D_{\mathrm{ps}}<0.25$ as the blue points - they represent the same particles, the only difference being the frequency of the rotating frames in which they close as 1:-1 (first), 2:-1 (second), 3:-1 (third) \& 3:-2 (fourth) orbits. The median frequency of each $\Omega_{m: l}$ distribution is plotted as the horizontal dashed line. Two separate groups appear at distinct frequencies. The larger group (with higher frequencies) is coincident with the 3:-2 LR and is in good agreement with the pattern speed of the bar as a family of 3:-2 orbits. The lower frequency group could be resonating in a 1:-1 fashion with the strong bar pattern or in a 3:-1 fashion with a weaker pattern at $\sim 22 \mathrm{~km} \mathrm{~s}^{-1} \mathrm{kpc}^{-1}$.

We also overplot the LR curves (blue/red) along with the CR curve (black) for the disc (the rotation curve is almost constant over the duration of the simulation so we use the curves as they are at the end of the simulation). The 1:-1, 2:-1, 3:-1 and 3:-2 LR are shown in the first, second, third \& fourth plots respectively. For the LR curves, we have used two different approaches corresponding to the blue and red lines. The red LR curves use $\kappa_{\mathrm{c}}$ which is evaluated from the epicyclic approximation using numerical differentiation. The blue LR uses the median $\kappa$ from the sample of resonant orbits along with $\Omega_{\mathrm{c}}$. For the outer (inner) LR, we use the median $\kappa$ of the orbits inside (outside) $11 \mathrm{kpc}$ (Note that these curves are only valid over the radius spanned by the blue points). Also, the particles generally rotate slower than indicated by the rotation curve meaning that $\Omega_{\mathrm{c}}$ is an overestimate of the true $\Omega$. This means that at a given radius the frequency of the LR is also slightly overestimated.

To begin with, we focus solely on the group with $\overline{R_{\mathrm{g}}}$ between 6 and $11 \mathrm{kpc}$. We look for coincidence of the blue points (the distributions of $\Omega_{m: l}$ ) with both a strong pattern (the red/yellow peaks in the spectrogram) and the location of a LR (the red/blue lines). We can see that the distributions of $\Omega_{m: l}$ (the blue points) only satisfy our assumptions above for the $m: l=3:-2$ orbit family (fourth plot). In this case, the guiding radii of the orbits are coincident with the 3:-2 LR and have almost the same pattern speed as the bar $\left(\sim 40 \mathrm{~km} \mathrm{~s}^{-1} \mathrm{kpc}^{-1}\right)$. This is good evidence that the group of periodic orbits inside 11 kpc are resonating in a 3:-2 fashion with the bar. The distributions of $\Omega_{m: l}$ are not coincident with any strong pattern as 1:-1, 2:-1 or 3:-1 orbits.

Similarly, for the group of orbits that lie outside 11 kpc, we see that they satisfy our assumptions in the $m=2$ spectrogram for the 1:-1 orbit family. First, as a family of 1:-1 orbits, they close in a rotating frame with almost the same pattern speed as the bar. Secondly, their location on the spectrogram coincides with the 1:-1 LR. There is also some agreement for the 3:-1 orbit family - a small feature lies inside the distribution with $\Omega_{\mathrm{p}} \approx 22 \mathrm{~km} \mathrm{~s}^{-1} \mathrm{kpc}^{-1}$. However, since the bar is much stronger than the pattern at $\sim 22 \mathrm{~km} \mathrm{~s}^{-1} \mathrm{kpc}^{-1}$, it is likely that these orbits are resonating in a 1:-1 fashion with the bar. Only a handful of particles inhabit this resonance $\left(\sim 1,750\right.$ for $D_{\mathrm{ps}}<0.04, \sim 2,300$ for $\left.D_{\mathrm{ps}}<0.06\right)$ and they make no significant contribution to kinematic structures in the disc (even though they make up $20 \%$ of all particles with $11<R_{\mathrm{g}}<15 \mathrm{kpc}$ for both cuts), so we do not consider them further. The 3:-2 orbits make up only $\sim 1 \%$ of all particles with $6<R_{\mathrm{g}}<10 \mathrm{kpc}$ for $D_{\mathrm{ps}}<0.025$ but increases to over $30 \%$ for $D_{\mathrm{ps}}<0.1$.

We remark that the measured frequencies $(\Omega$ and $\kappa)$ are estimates of the true frequencies. They do however serve well as a diagnostic and remove any ambiguity regarding the forcing resonant perturber. By estimating the frames $\left(\Omega_{m: l}\right)$ in which our sample of resonant orbits close as different orbit types, we can ignore families for which there are no patterns with commensurate frequencies. For example, we see that for the 2:-1 orbit family (second plot), there are no patterns that could force this type of resonance and so we disregard it. The best agreement lies with the bar pattern being the forcing perturber for the 3:-2 and 1:-1 orbit families.

\section{RESONANT ORBIT DYNAMICS}

Our sample of resonant orbits represents kinematic substructure in the disc that is not present in an axisymmetric potential. The choice of a cut on the minimum phase space distance may seem arbitrary and does introduce uncertainty on the fraction of particles on resonant orbits. However, it does demonstrate the types of resonances that are populated and can persist for a significant duration in discs with a strong central bar. By extracting a sample of resonant orbits from N-body simulations, we can also look more closely at their behaviour as the disc evolves. Here, we concentrate on resonances beyond CR but this method works just as well for the bar orbits.

\subsection{Resonant Clumping}




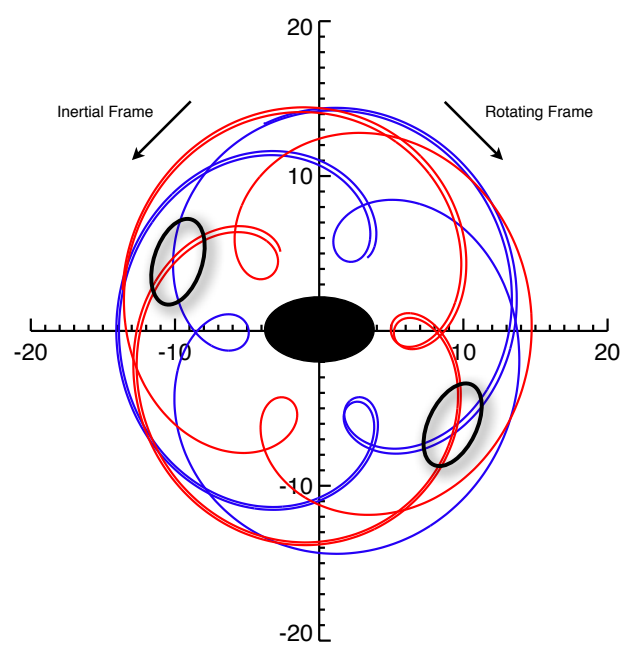

Figure 5. Possible orientations of the 3:-2 orbit, which has twofold symmetry with respect to the bar aligned with the $x$-axis. We define a chirality so the the orientation with a pericentre on the negative $x$-axis is left-handed (blue) and that with a pericentre on the positive $x$-axis is right-handed (red). The orientations of the 3:-2 orbit family allow six possible pericentres (and apocentres). When we overplot the two orientations, we can identify locations (with respect to the bar) at which there are both inward and outward moving groups (indicated by the black ovals). In the inertial frame, the bar and particles rotate in an anti-clockwise fashion. In the rotating frame with $\Omega=\Omega_{b a r}$, the bar remains aligned with the $x$-axis and the particle move in a clockwise fashion. The locations occur on the trailing side of the bar at longitudes similar to the Solar position.

Our sample of 3:-2 orbits behave in a highly coordinated fashion. They are not distributed evenly around the orbit, but instead exist in multiple populations that vary in their epicycle and azimuthal phase. In the following, we describe populations that are in phase in epicycle as groups. Within these groups are populations with the same azimuthal phase which we call subgroups. With this nomenclature, the groups all reach their peri-/apocentres at the same time, while the subgroups have their peri/apocentres at different azimuths. Figure 5 shows the two possible orientations of the $3:-2$ orbit - each orientation has one pericentre at either end of the bar which is aligned with the $x$-axis and the orbits move in a clockwise fashion (in the inertial frame the disc rotates in an anti-clockwise fashion). Since we have defined $l$ to be negative for orbits beyond CR, Equation 10 means that $\Omega_{m: l}$ is always larger than $\Omega$, the orbital frequency in the inertial frame (at least for orbits with $\kappa>0$ ). The orbital frequency in the rotating frame, $\Omega^{\prime}$, is then necessarily negative, i.e.,

$$
\Omega^{\prime}=\Omega-\Omega_{m: l}<0,
$$

so that in the rotating frame the orbit moves in an opposite sense to the inertial frame. It is useful here to define a chirality, or handedness, for each orientation. Those orbits that have one of their pericentres on the negative $x$-axis are left-handed (i.e., on the left end of the bar - the blue orbit), while those with one on the positive $x$-axis are right-handed (the red orbit).

If the particles populating these types of orbits are distributed uniformly in azimuth, then we should see bi-

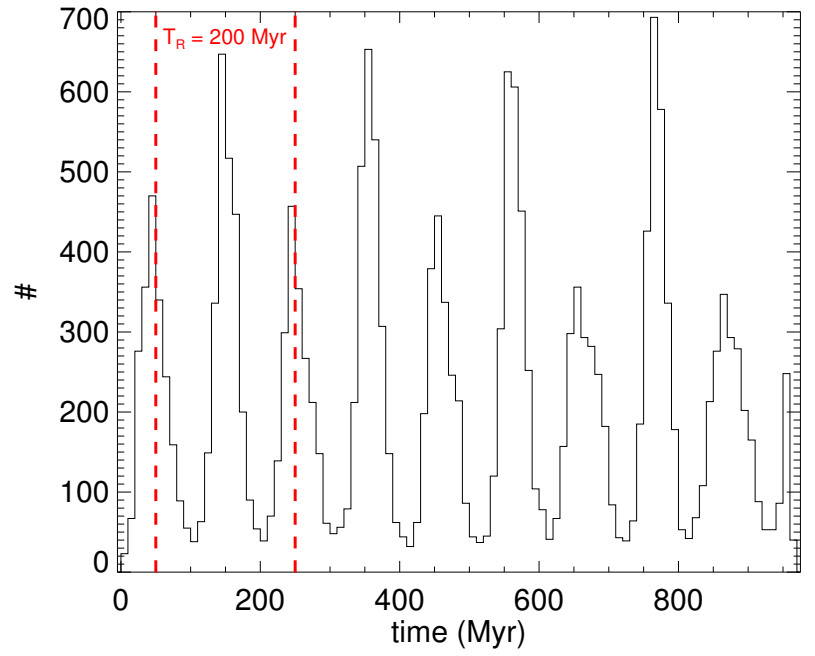

Figure 6. The distribution of times at which our closed orbits reach their pericentres (over the last $0.96 \mathrm{Gyr}$ ). Note that the orbits mostly conspire to reach their pericentres at roughly the same time. This is the phenomenon of resonant clumping in the phase angle $\theta_{R}$ (where $\dot{\theta}_{R}=\kappa$ ). We indicate the epicyclic period, $T_{R}$, for our sample of 3:-2 closed orbits as the vertical red lines. Peaks occur on a timescale of one half of $T_{R}$ which means our closed orbits are made up of two groups whose epicyclic motion is out of phase by $\pi$.

modal $v_{R}$ distributions at a number of discrete angles with respect to the bar. This is not the case, however. When we measure the times at which the particles reach their pericentres, we see that they are arranged in such a way as to reach their pericentres at almost the same time. Figure 6 shows the distribution of times at which our closed 3:- 2 orbits pass through their pericentres. This is clear evidence that the particles are not uniformly distributed and so we expect a resonant clumping of the particles in azimuth as all of the particles coherently reach their pericentres. It is also clear that the $3:-2$ resonant orbits are split into two distinct groups whose epicycles are out of phase by $\pi$. The median $\kappa$ for the 3:-2 orbits is $\approx 30 \mathrm{~km} \mathrm{~s}^{-1} \mathrm{kpc}^{-1}$, which equates to an epicyclic period of $T_{R} \approx 205$ Myr. Figure 6 shows that, as one group of 3:2 orbits reaches pericentre, the other reaches apocentre. The groups then pass each other as they each complete their epicyclic motion - i.e., they comprise of an outward moving group and an inward moving group. This coordinated movement gives rise to transient overdensities at pericentre and apocentre and to bimodal distributions in $v_{R}$.

We have checked that this phenomenon is not some artefact introduced by our use of the phase space distance method. By estimating $\Omega$ and $\kappa$ for a random sample of $10 \%$ of the simulation particles, we extract an independent sample of 3:-2 periodic orbits. This sample also exhibits clumping on timescales consistent with the epicyclic period and confirms that no bias towards certain epicyclic phases is introduced.

Each group is host to a number of subgroups. When a group reaches pericentre, its constituent subgroups populate over-densities which occur at different azimuths. The preferred azimuths have distinct locations with respect to the bar - the allowed locations correspond to the six pericentres shown in Figure 5 (three red and three blue). It is not necessary for all six locations to be pop- 


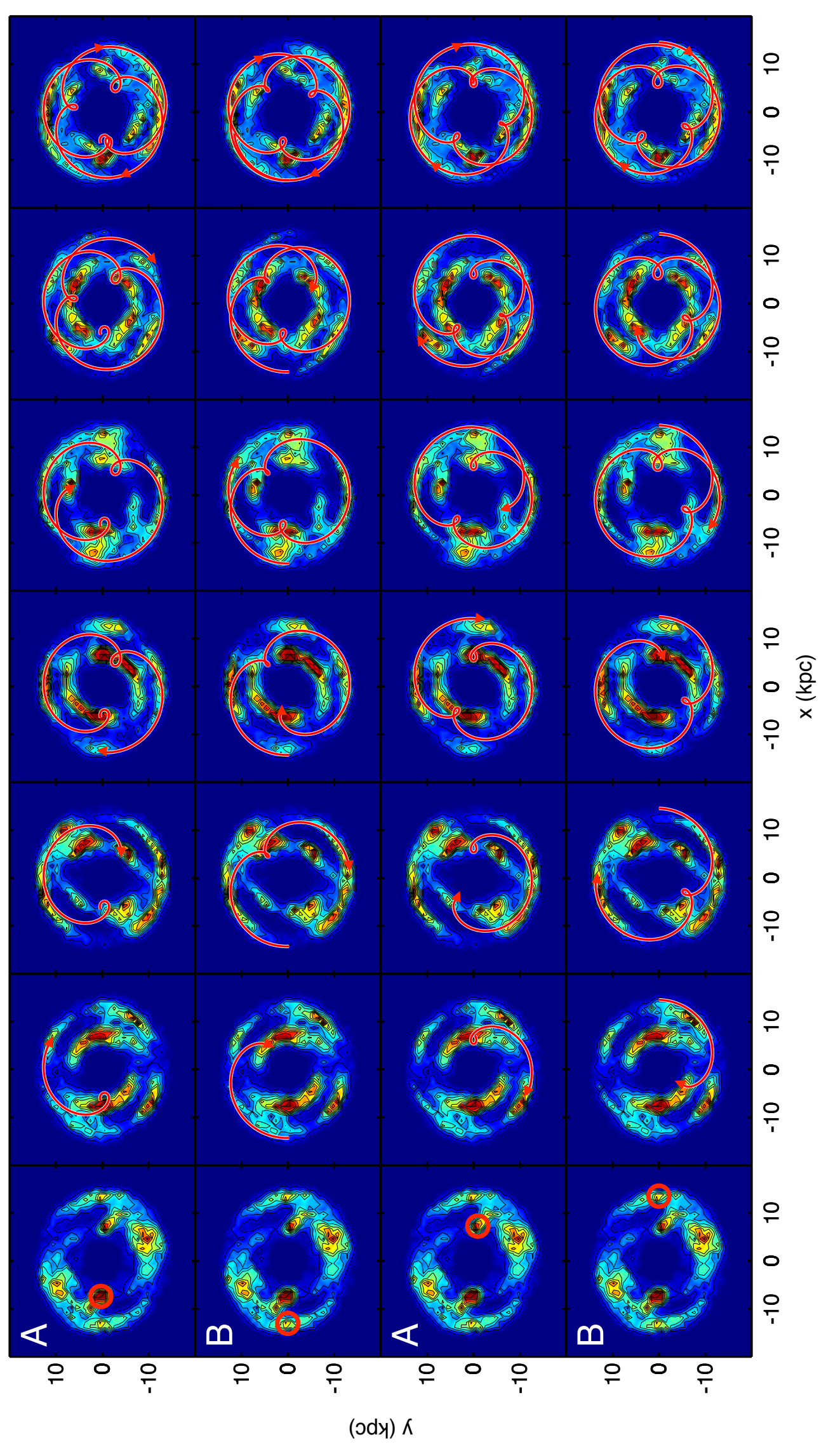

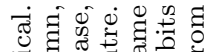

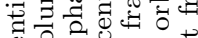

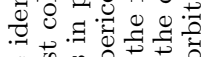

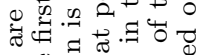

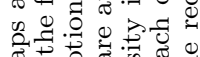

घ $\Xi$ :

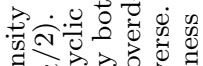

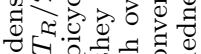

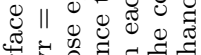

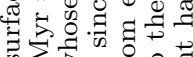

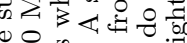

다웅

दै

כ)

ن

¿

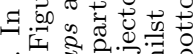

.

대

\& 00 o

+4 崖

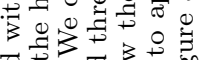

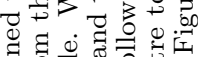

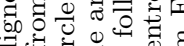

ब

O

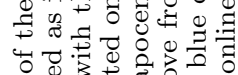

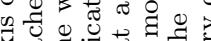

政 0 \%

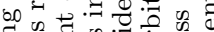

워.

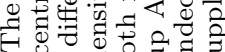

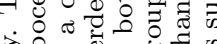

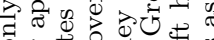

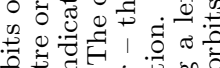

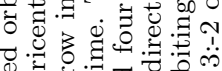

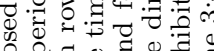

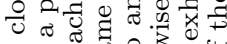

N \& व

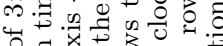

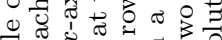

चี

w

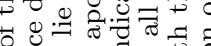

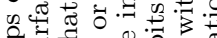

胥

द्व.

की

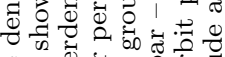

ญ

क्ञ

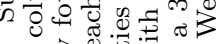

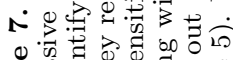

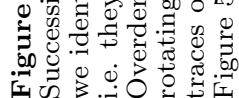




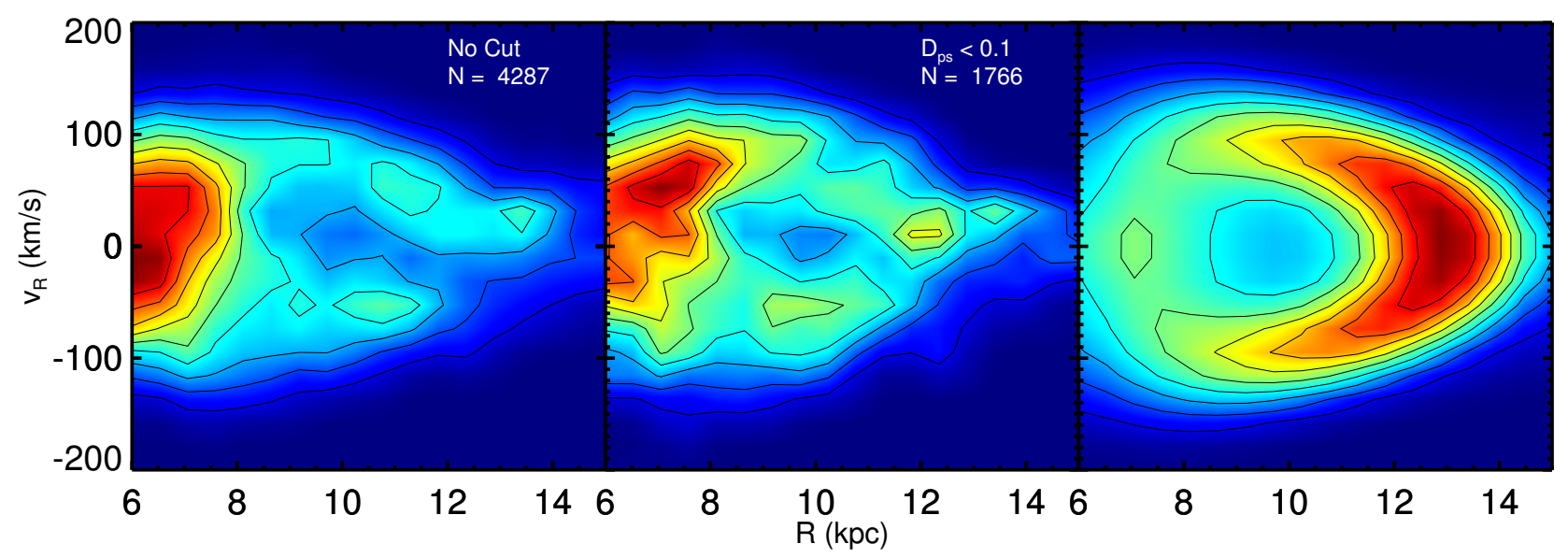

Figure 8. The distribution of $v_{R}$ as a function of $R$ for a pencil beam towards the anti-Centre at 4.8 Gyr. The number of particles $N$ is also indicated. The disc is rotated so that the bar is at an angle of $20^{\circ}$ with respect to the $x$-axis. Particles for which $|z| \leq 0.5 \mathrm{kpc}$ and $|y| \leq 1 \mathrm{kpc}$ make up the distributions. On the left, we show the distribution for all particles. A strong kinematic feature is present, namely the bimodal distribution of $v_{R}$ between 10 and $12 \mathrm{kpc}$. The middle plot shows the distribution generated by only the resonant orbits using a cut on the phase space distance of $D_{\mathrm{ps}}<0.1$. The right plot shows the time-averaged imprint of the resonant orbits in $R$ - $v_{R}$ space. The density in this plot is a measure of the time spent at each location, meaning that the persistence of kinematic features scales with the radius at which they occur.

ulated but, in order to avoid lopsidedness, if one is populated then another on the opposite side must also be populated with a similar number of particles. We follow these coherent motions by plotting the surface density of the 3:-2 resonant orbits at times when a peri/apocentre is reached (Figure 7). The surface density maps in each column are identical and are plotted when a peak occurs in the distribution of pericentre times in Figure 6 . In each map, the bar is aligned with the $x$-axis. In the first column, we see four overdensities that lie along the $x$-axis - two inner and two outer marked by the red circles in each row. The inner overdensities (with $R \approx$ $8 \mathrm{kpc}$ ) correspond to a single group, labelled group A. Each (inner) overdensity then corresponds to a subgroup of group A. These subgroups are in-phase with respect to their epicycles but are exactly $\pi$ out of phase in their azimuthal motion. Similarly, the outer overdensities on the $x$-axis (with $R \approx 12 \mathrm{kpc}$ ) constitute group $\mathrm{B}$, whose epicycles are out of phase by $\pi$ compared to group A. As with group A, group B is host to subgroups - those subgroups on the $x$-axis are also $\pi$ out of phase in azimuth. So, to reiterate, two overdensities in group A are denoted by the red circles in rows one and three, with both rows indicating separate subgroups. Rows two and four indicate two of the overdensities from group B with, again, each row indicating separate subgroups. For each separate subgroup, we choose a particle and follow its trajectory through successive apo/pericentres. In all cases, the bar is aligned with the $x$-axis and the orbits progress in a clockwise direction.

In the second column, the particles from group A have moved from pericentre to apocentre, and vice versa for group B. At some intermediate point, the particles from each group have passed each other, with group A having positive $v_{R}$ and group $\mathrm{B}$ having negative $v_{R}$. This passage generates a bimodal distribution of $v_{R}$ in the regions of space where the two groups pass each other. As we move to the third column, group A has returned to pericentre while group B has moved again to apocentre. Each successive column indicates a peri- or apocen- tre until the final column, where the 3:-2 orbit has been completed. In terms of chirality, each group is host to both left- and right-handed 3:-2 orbits (the top two rows are left-handed and the bottom two right-handed). Note also that for this simulation, each group is host to four out the six possible subgroups. This is evidenced by the absence of an overdensity at the end of the bar in every third snapshot. If all six subgroups were populated, then each time a pericentre is reached, an overdensity would appear at the ends of the bar.

The evolution of these distinct orbital groups and subgroups leads to a "pulsing" of the density distribution in the disc with stars periodically and coherently moving inwards and outwards. These transient overdensities contribute to the time-dependent, but periodic, nature of the galactic potential. The presence of these two populations contributes to stabilizing what seems to be an inherently lop-sided configuration. This mechanism of coherence among the resonant orbits suggest that strong kinematic features can be generated as inward and outward moving groups encounter each other.

\subsection{Kinematic Signatures}

This bimodal distribution of $v_{R}$ constitutes a potentially observable feature from the Solar position. At some point, overdensities appear in line with the long axis of the bar on one side of the disc, one due to an accumulation at pericentre and one due to an accumulation at apocentre (one from groups $\mathrm{A}$ and B; e.g., rows $1 \& 2$, Figure 7). As they both progress to the extremes of their respective epicycles, the bar moves ahead in azimuth. This means that they pass each other on the trailing side of the bar, generating a bimodal distribution in $v_{R}$. This bimodal distribution appears at distinct azimuths - specifically, between the allowed locations for the pericentres and apocentres (see Figure 5).

The angle of the bar with respect to the line joining the Sun and Galactic Centre is poorly constrained. It is estimated to be in the region of $10^{\circ}$ to $40^{\circ}$ (e.g., Stanek et al. 1997; Freudenreich 1998; Robin et al. 2012; 
et al. 2013, Cao et al. 2013). For an angle of $\sim 20^{\circ}$ on the trailing side of the bar, there is a bifurcation in the radial velocities between 10 and $11 \mathrm{kpc}$. We indicate these regions with the black ovals in Figure 5. We can simulate a field of view towards the anti-Centre and measure the distribution of $v_{R}$ as a function of galactocentric distance $R_{\mathrm{GC}}$. By doing this for only the resonant orbits, we can see how the bimodal nature of the $v_{R}$ distribution emerges (Figure 8). If we rotate the disc so that the bar is at an angle of $20^{\circ}$ with respect to the $x$-axis and select only particles that have $|y| \leq 1 \mathrm{kpc}$ and $|z| \leq 0.5 \mathrm{kpc}$, a bimodal distribution of $v_{R}$ is present between 10 and 11 kpc. This feature is still significant when we consider all (i.e. resonant and non-resonant) particles (left, Figure 8).

This result is in good qualitative agreement with the recent observations of Liu et al. (2012) who, for a sample of Red Clump stars in a pencil beam towards the Galactic anti-Centre, find a bimodal distribution of heliocentric (line-of-sight) radial velocities, $v_{\text {los }}$. The distribution of radial velocities has two significant peaks falling in the range $10<R_{\mathrm{GC}}<11 \mathrm{kpc}$ at $v_{\text {los }}=-4 \mathrm{~km} \mathrm{~s}^{-1}$ and $v_{\text {los }}=+27 \mathrm{~km} \mathrm{~s}^{-1}$. Since the field is directed towards the anti-Centre, the line-of-sight velocities $v_{\text {los }}$ serve as a good proxy for the galactocentric radial velocities, $v_{R}$. For the anti-Centre, we have

$$
v_{\mathrm{GSR}}=v_{\mathrm{los}}-\mathrm{U} \approx v_{R}
$$

By correcting for the radial Solar motion, which has an inward motion of $10 \lesssim \mathrm{U} \lesssim 14 \mathrm{~km} \mathrm{~s}^{-1}$, the distribution becomes symmetric about $v_{G S R}=0 \mathrm{~km} \mathrm{~s}^{-1}$ with peaks at roughly $\pm 15 \mathrm{~km} \mathrm{~s}^{-1}$.

Liu et al. (2012) suggest that this feature is due to a resonant interaction with the Milky Way's bar, since their locations are consistent with the position of the Outer Lindblad Resonance. The bimodal distribution in our simulation occurs outside the bar's OLR $(\sim 8.5 \mathrm{kpc})$. Since our disc is kinematically hot $\left(\sigma_{v_{R}} \approx 65 \mathrm{~km} \mathrm{~s}^{-1}\right.$ at $R=9 \mathrm{kpc}$ ), the difference between the two peaks in the velocity distribution is larger than the observations. We have however provided a possible mechanism for such a feature to arise. If the resonance is populated in a hot disk, then it is likely to be still more important in a colder disk. In hotter disks, the larger velocity dispersions of stars means they are less likely to become and remain trapped in narrow resonances.

Finally, in the right plot of Figure 8, we show the timeaveraged imprint of the 3:- 2 orbits in $R-v_{R}$ space. The vertical and horizontal extent of the distribution is a measure of the epicycle energy associated with the orbits and the density can be thought of as a measure of the time spent by the particles in these regions. The particles spend little time at pericentre with the majority of the orbit spent at large $R$. Kinematic structures due to the resonant orbits are therefore more persistent if they occur in the outskirts of the disc.

\section{DISCUSSION}

To our knowledge, there has been little investigation of resonant orbits in the outskirts of barred N-body discs. Resonant clumping therefore seems to be a previously unknown phenomenon. For this reason, we discuss the mechanism that cause the periodic orbits to become clumped in phase angle $\left(\theta_{R}\right)$ and to remain so for long periods in the evolution in the disc. In the following, we address two questions: (1) How do the periodic orbits become clumped in the first place? and (2) Why do they remain clumped when phase mixing should work to undo the clumping?

\subsection{Resonant Clumping: Origins}

To investigate the first point, we apply the phase space distance method described in Section 2 over many epochs during the evolution of the disc. Specifically, we use eight $\sim 1$ Gyr time segments, from $\sim 0.5$ Gyr to $\sim 1.5 \mathrm{Gyr}, \sim 1.0$ Gyr to $\sim 2.0$ Gyr, 1.5 Gyr to $\sim 2.5$ Gyr all the way up to $\sim 4.0$ to $\sim 5.0 \mathrm{Gyr}$, which was the segment we analysed in the previous sections of the paper. We avoid using data from the epoch of bar formation (0-500 Myr), since angular momentum is violently redistributed throughout the disc. The resulting variations in the guiding radii and rotational speeds make the normalisations on the phase space distance unreliable. In any case, we are able to extract resonant orbits from all eight segments. The number of resonant orbits increases with time and begins to flatten out after segment five (i.e, from $\sim 2.5 \mathrm{Gyr}$ onwards). We also find, by analysing the azimuthal and radial frequencies, that the 3:-2 orbit family is the dominant family even from early on.

For our current purposes, we are interested in particles that are captured into resonance early on and that remain in resonance for a long time. To select such a long-lived resonant sample, we take particles found during segment two ( $\sim 1$ Gyr to $\sim 2$ Gyr) and compare them to the particles found during segment eight ( $\sim 4$ Gyr to $\sim 5$ Gyr). Particles that are common to both samples have remained in resonance for at least $\sim 4$ Gyr.

Due to the changes in angular momentum (and the corresponding changes in $R_{\mathrm{g}}$ and $\Omega$ ), especially during the formation of the bar, a reliable measure of the phase angle $\theta_{R}$ (i.e., the clumped angle) is unavailable to us. For this reason, we continue to use the times at which the particles reach pericentre as an indicator of whether clumping in the phase angle is present or not. While this is a robust indication of the presence of clumping, we are unable to say if the particles become clumped in the time period between apocentre and pericentre. In Figure 9 (top panel), we show the distribution of pericentre times for the 3:-2 particles extracted from segment two ( $\sim 1$ Gyr to $\sim 2$ Gyr; black) and the 3:-2 particles that are common to segment two and segment eight (the long-lived resonant particles; blue). The distributions are shown from $t=0$ Gyr to $t=\sim 2$ Gyr and indicate that the particles become clumped during the formation of the bar (between $200 \mathrm{Myr}$ and $500 \mathrm{Myr}$ ).

In the bottom panel of Figure 9, we show the surface density of all particles in the disc (top row) during the formation of the bar with contours of equal density. The bar in this case forms very quickly (within $300 \mathrm{Myr}$ ) and its arrival induces strong spiral structures. We also show the surface density for our sample of 3:-2 particles from segment two in the bottom rows, this time with contours of equal effective potential. The outline of the resonant orbits exhibits a striking resemblance to the large ringed-shaped features seen in external barred galaxies (the so-called ' $\mathrm{R}$ ' rings of Buta (1986)). It is also clear that overdensities appear close to the Lagrange points at either end of the bar (i.e., $\mathrm{L}_{1}$ and $\mathrm{L}_{2}$ ). Given that (a) the periodic orbits are clumped in $\theta_{R}$ as the bar is forming, (b) the bar induces strong spirals, (c) the resonant particles appear to follow the pattern of rings in barred galaxies and (d) overdensities appear close to the Lagrange points, we naturally suspect that the clumping 

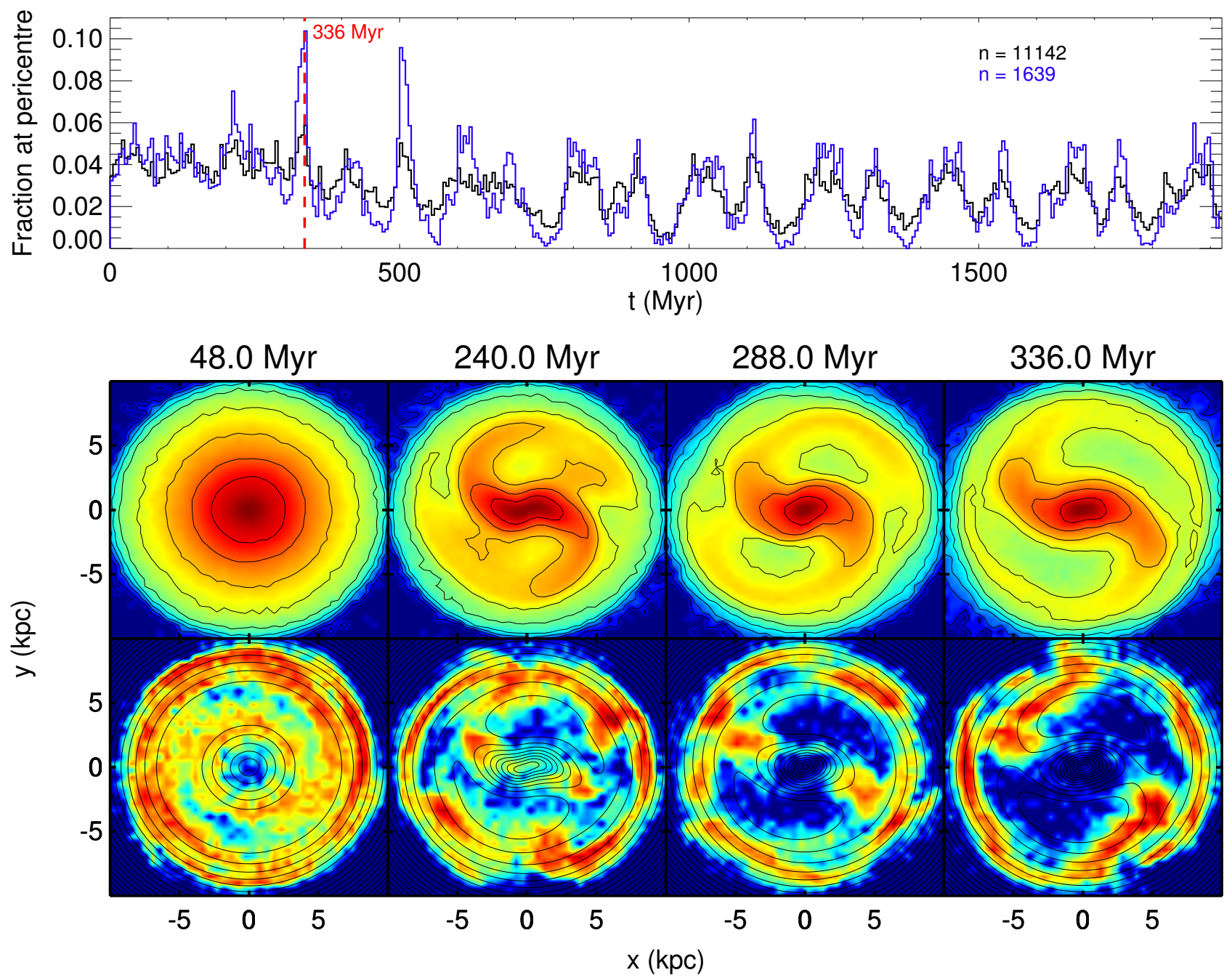

Figure 9. Clumping occurs early on in the evolution of the disc. In the top panel, we show the distribution of pericentre times for particles extracted from segment two (black) and particles common to segments two and eight (blue). The surface density of the disc during the formation of the bar is shown in the top row of the bottom panel (contours of equal surface density). The resonant particles exhibit overdensities at the Lagrange points at either end of the bar (contours of equal effective potential, as shown in the final row).

is related to the mechanism that generates these type of structures - namely, passage through the unstable Lagrange points and their associated manifolds.

The mechanics of bar induced spirals and large scale rings in barred galaxies has been studied in detail (cf. Gómez et al. 2004, Romero-Gómez et al. 2006, 2007. Athanassoula et al. 2009a b). The first main point relevant to our work is that $L_{1}$ (and $L_{2}$ ) are host to the Lyapunov orbits (roughly elliptical orbits that corotate with $\left.L_{1 / 2}\right)$. Since the region is unstable, the periodic Lyapunov orbits can't indefinitely trap other regular orbits. Orbits that visit this region tend to leave it on a timescale proportional to their energy, i.e., lower energy orbits escape the region faster than orbits with a higher energy. The second main point is that the trajectories through which the orbits (which may have been temporarily trapped) can leave this region are determined by the unstable manifolds - whereas the stable manifolds describe the trajectories through which orbits approach this region. As an example, we plot some orbits that follow these manifolds in Figure 10. Note that not all of the resonant orbits approach the Lagrange points. From the total sample of resonant particles, we estimate that about $75 \%$ approach the Lagrange points. For these, the average radius at $t=0 \mathrm{Myr}$ is $R_{0} \approx 4 \mathrm{kpc}$ with $2<R_{0}<7 \mathrm{kpc}$. In the left (right) panel, we show orbits that come from the inner (outer) parts of the disc and in the right. At $t \approx 150 \mathrm{Myr}$, the majority of particles have $R<4 \mathrm{kpc}$ indicating passage along the inner stable manifold (i.e., along the bar). The green crosses indicate the positions corresponding to the time at which the surface density and equipotential contours are plotted. The orbits can be compared with those shown in Figure 4 of Athanassoula (2012).

'The third, and possibly most important, point in this context is that the $\mathrm{L}_{1}$ and $\mathrm{L}_{2}$ points can be stabilised by concentrations of matter (see section 5 of Athanassoula et al. 2009b). This generates a local minimum in the potential, a stable equilibrium point surrounded by two unstable saddle points (each with their own manifolds). This stable minimum can trap particles and keep them in the vicinity for a long period of time. As more particles gather here, so the minimum in the potential deepens, and the overdensity grows. This seems a plausible mech- 


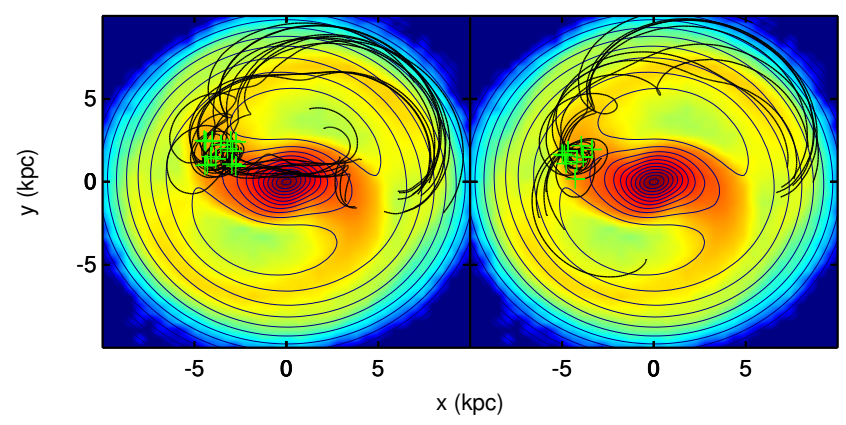

Figure 10. The surface density of all particles at $\sim 200 \mathrm{Myr}$ with contours of equal effective potential $\Phi_{\text {eff. }}$ Left panel: Trajectories of periodic orbits that approach $\mathrm{L}_{1}$ from the bar. Right panel: Trajectories of periodic orbits that approach from the outer disc. The orbits are plotted from $\sim 100$ to $450 \mathrm{Myr}$. The green crosses indicate the positions at $\sim 200 \mathrm{Myr}$. The particles move in a clockwise direction (in the frame corotating with the bar).

anism for the initial clumping.

We have shown that at the epoch of bar formation, the phase angles are clumped, as they appear to reach pericentre at the same time (Figure 9. top panel). We have also shown that the orbits pass close to the unstable Lagrange points on either end of the bar (Figure 9 bottom panel) and as they exit they follow the unstable manifolds (Figure 10) and exhibit a structure similar to the manifold-driven spirals. Since we can only probe the clumping at apo/pericentre, we can't demonstrate whether the clumping in the phase angle occurs before, during or after the particles move through the Lagrange points. So, while we can only speculate as to the mechanism by which the phase angles become coherent - due to passage through the unstable equilibrium points - we can conclusively say that the initial clumping occurs during the formation of the bar. A rigorous investigation of the mechanism that causes the clumping of the phase angles requires an experiment in which the phase angles can be measured at all points along the orbit.

\subsection{Resonant Clumping: Persistence}

Since the clumping in the phase angle $\theta_{R}$ occurs very early on, it is natural to expect that, as the disc evolves, the phase angle becomes mixed and the clumping should become less prominent. This is not the case however. For our sample of long-lived resonant particles (from the blue histogram, top panel, Figure 9), we see that the clumping in their phase angles persists for nearly $4 \mathrm{Gyr}$, apparently becoming more coherent as time progresses. The top plot in Figure 11] shows the distribution of pericentre times for this sample. The clumping occurs on timescales comparable with the radial period of the epicycle for these particles (with $\kappa \approx 30 \mathrm{~km} / \mathrm{s} / \mathrm{kpc}, T_{R} \approx 205 \mathrm{Myr}$ ) and remains so for almost the whole duration of the simulation. This is clear evidence that something is counteracting the effects of phase mixing.

Since the phenomenon of resonant clumping necessarily involves transient overdensities, we suggest that changes in the actions $J_{R}$ and $J_{\phi}$ are working against phase mixing and keeping them locked in phase angle. Below we set out how we measure the actions. Note we do this only for the particles caught in segments $2 \& 8$ that are deemed to be in a 3:-2 resonance - i.e., those from the top panel of Figure 11 .

We compute the actions $(J)$ by numerically integrat- ing over the N-body trajectories. We measure the radial action $J_{R}$ as

$$
J_{R}=\frac{1}{\pi} \int_{R_{\min }}^{R_{\max }} v_{R} d R
$$

where we have integrated over half of the epicycle. The next measurement is taken over the second half of the epicycle. To follow the evolution of the actions, we assign a time to each measurement. If we begin the measurement at $t\left(R_{1}\right)$ and end it at $t\left(R_{2}\right)$ with the duration $\triangle t=t\left(R_{2}\right)-t\left(R_{1}\right)$, then we say that orbit has an action $J_{R}$ at time $t=t\left(R_{1}\right)+\Delta t / 2$. It is also convenient to measure the epicyclic frequency $\kappa$ as

$$
\kappa=\frac{\pi}{t\left(R_{2}\right)-t\left(R_{1}\right)} .
$$

We measure the azimuthal action as

$$
J_{\phi}=\frac{1}{\pi} \int_{\phi_{1}}^{\phi_{2}} L_{z} d \phi
$$

where $\phi_{1}$ and $\phi_{2}$ are the azimuths at which pericentre or apocentre is reached, i.e., the azimuths at $t\left(R_{1}\right)$ and $t\left(R_{2}\right)$ from above. In a similar way to our measurement of $\kappa$, we reckon $\Omega$ as

$$
\Omega=\frac{\phi_{2}-\phi_{1}}{t\left(R_{2}\right)-t\left(R_{1}\right)} .
$$

The fast action $J_{\mathrm{f}}$ is just a linear combination of $J_{R}$ and $J_{\phi}$ (see Collett et al. 1997, for details), so that

$$
J_{\mathrm{f}}=J_{R}+\frac{|l|}{m} J_{\phi}
$$

where $m \& l$ are the usual integers describing the closed orbit. $J_{\mathrm{f}}$ describes the motions of particles along the orbit pattern while the slow action, $J_{\mathrm{s}} \approx J_{\phi}$, describes the precession of the apsides of the orbit pattern (the 3:-2 pattern).

Figure 11 (second panel) shows the evolution of the fast actions for the sample of resonant particles extracted from time segments $2 \& 8$. The combination of slightly increasing radial actions and slightly decreasing azimuthal actions (not shown) leads to a distribution of fast actions that barely changes. We have also made an estimate of $\triangle J_{\mathrm{f}}$ and $\triangle \kappa$, by taking one measurement of $J_{\mathrm{f}}$ or $\kappa$ subtracted from the next. We remark that this is not the time derivative of $J_{\mathrm{f}}$, but the change per half epicycle and gives us an idea of the trends in the actions and frequencies. Figure 11 (third panel) shows that although the overall distribution of $J_{\mathrm{f}}$ does remain largely constant, there are changes in the actions of individual particles. The changes are most pronounced at earlier times, but persist until the later stages of the disc's evolution. Figure 11 (fourth panel) then shows that the changes in $J_{\mathrm{f}}\left(\triangle J_{f}\right)$ are accompanied by changes in $\kappa(\triangle \kappa)$. This is exactly the same as Figure 11 (third panel) except instead of colour representing density, it now represents the average change in $\kappa$-blue represents a decrease in $\kappa$ and red represents an increase. If, for a particular particle, the fast action has increased, then this is accompanied by a decrease in the corresponding frequency - which is $\kappa$ in this case. The opposite occurs for a decrease in $J_{\mathrm{f}}$. The distributions of pericentre times shows that mixing in the phase angle is not occurring, so that the changes in the frequency $\kappa$ work against the phase mixing. 


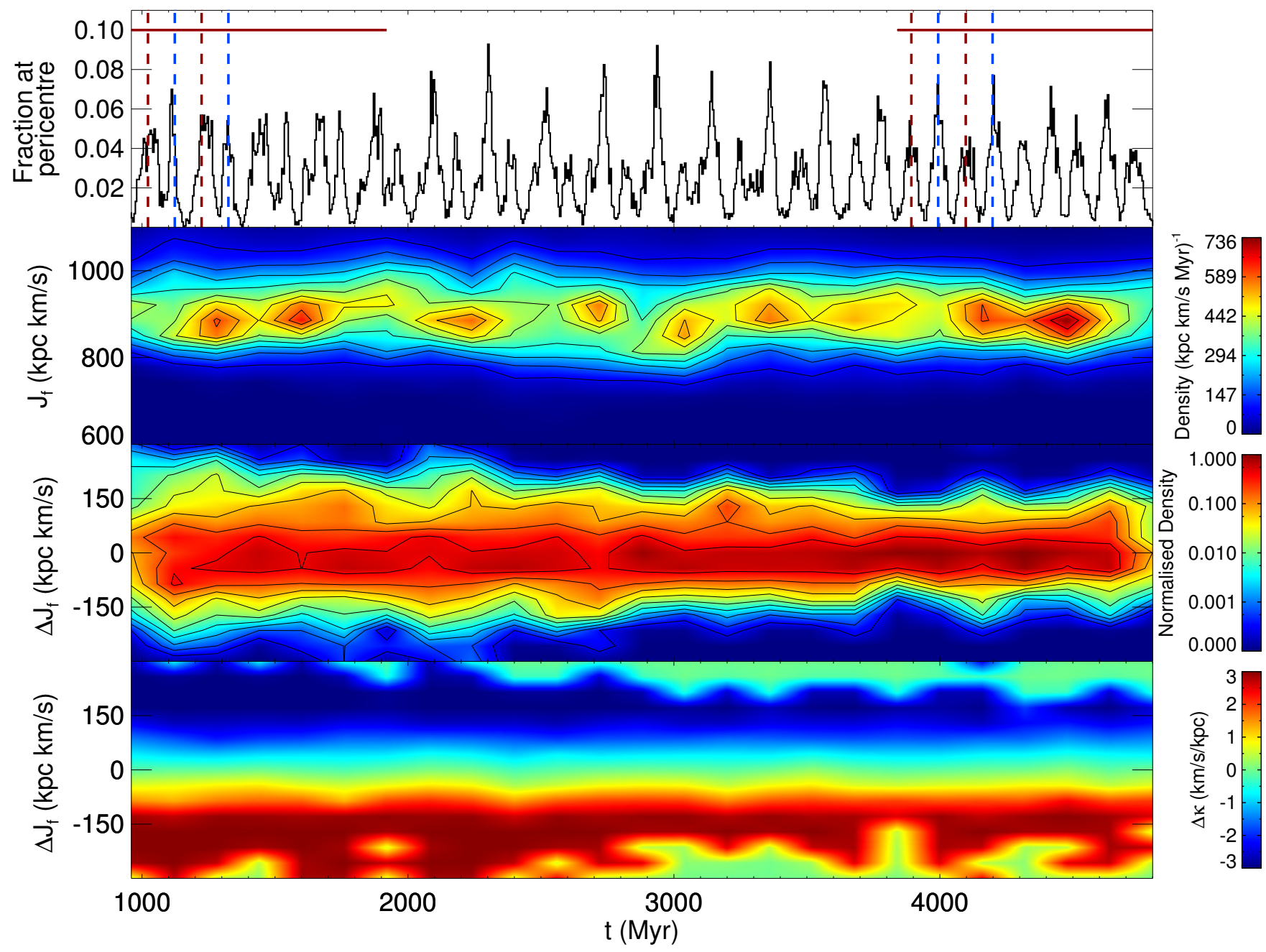

Figure 11. Phase mixing is inefficient, as the clumping in the phase angle $\theta_{R}$ persists for long periods. The pericentre times are shown for the resonant particles common to segments two and eight (highlighted with red on the horizontal axis). The overall distribution of fast actions $\left(J_{\mathrm{f}}\right.$ second row) remains unchanged over the $\sim 4$ Gyr shown here. Individual particles do however experience changes in their respective actions shown by the distribution of $\triangle J_{\mathrm{f}}$ in the third panel. Changes in the fast action are accompanied by changes in the corresponding frequency $\kappa$ (where $\dot{\theta}_{f}=\dot{\theta}_{R}=\kappa$ ).

\section{SUMMARY \& CONCLUSIONS}

With the emergence of N-body simulations as the workhorse in the study of galaxy dynamics and evolution, it is important to have the tools available to extract as much information as possible from them. The potential in galaxy discs is inherently time-dependent, driven by evolving bars, rings and spiral patterns. This then prevents a steady state description of the disc using a time-independent Hamiltonian. Frequency analyses also encounter issues in highly dynamic, unsteady and evolving systems. When low frequency orbits occur in varying potentials, it can be hard to separate them from induced radial modes (e.g., Ceverino \& Klypin 2007). In discs with significant angular momentum exchange, it can also be difficult to measure the characteristic frequencies of the orbits $(\kappa$ and $\Omega$ ).

Resonant orbits play a special role in such systems. We have developed a method to make rapid and automated searches for closed orbits in N-body simulations. This al- gorithm utilises the fact that orbits resonating with a bar or spiral pattern in a rotating frame return periodically to previously inhabited spot in phase space. By defining a metric that measures distance travelled in phase space, we have extracted samples of closed orbits from the simulation of Shen et al. (2010). The sample can be tuned by use of an arbitrary phase space distance cutoff point. The choice of cutoff depends on how "clean" a sample of closed orbits is required. This essentially separates an Nbody simulation into distinct components - the, possibly many, resonant families and the background disc population. The method has its greatest utility in complex dynamical systems such as a Galactic bar where perturbation theory and frequency analysis may have difficulty (see e.g., Molloy et al 2014b).

As a worked application, we use the method to dissect the N-body simulation of Shen et al. (2010). This starts as an unstable disc that rapidly forms a massive bar, which evolves through buckling instabilities to give a bulge that is an excellent match to the data in the cen- 
tral parts of the Milky Way. However, a drawback to the simulation is that the outer disc is kinematically hotter than is the case for the Milky Way, which prevents a direct comparison with data. The size of the bar and the absence of both gas and a live halo mean that angular momentum exchange in the disc is maximised. These two aspects of the simulation mean that perturbation theory and a frequency analysis would rapidly encounter their respective limitations.

In the outer disc, we extracted a sample of resonant orbits. By combining the orbital properties of our sample with Fourier spectrograms, we have demonstrated the source of the resonance as the central bar itself (Figure 4). In this simulation, the major populations of resonant orbits are the 3:-2 and 1:-1 families. By measuring the epochs at which closed orbits reach their turning points, we see that they move in a coordinated fashion. The particles that populate these resonant orbits all conspire to reach their pericenters and apocentres at roughly the same time (Figure 7). This leads to the phenomenon of resonant clumping in the disc. Overdensities are produced each time a pericenter or apocentre is reached. These overdensities can make a significant contribution to the disc potential and their transient nature prohibits a description of the disc by means of a time-independent Hamiltonian.

The family of 3:-2 orbits exist in two distinct groups, whose epicycles are out of phase by $\pi$. This means that as one group reaches apocentre, the other reaches pericentre. As the particles complete their respective epicyclic motions, they overlap in configuration space with one group moving inwards, and the other moving outwards. This characteristic of their collective motion generates a bimodal distribution of galactocentric radial velocities that occurs at distinct angles with respect to the bar. For the family of 3:-2 orbits, this occurs at angles of between $20-40^{\circ}$ which is in the range measured for the viewing angle of the Galactic bar. While we refrain from making a direct comparison with the Milky Way due to the hotness of the simulated disc, we have provided a mechanism which may explain the bimodal distribution of $v_{R}$ observed by Liu et al. (2012) towards the anti-Centre.

Each group can also be divided into subgroups depending on the azimuth at which they reach their pericenter or apocentre. For example, if one subgroup reaches their pericentre on the negative $x$-axis (i.e., at one end of the bar), another subgroup appears on the positive $x$-axis. This means that, as one overdensity is produced, so another is produced on the opposite side of the disc. This maintains a bisymmetric pattern and prevents the disc from becoming lopsided.

The phenomenon of resonant clumping has not received much attention hitherto. For this reason, we have endeavored to uncover how it comes about, and why it persists in the disc. The global morphology in the density distribution of the resonant particles along with their proximity to the unstable Lagrange points (and their approach and departure) suggests there is a strong link between the initial clumping mechanism and that which brings about bar-induced spirals - namely, passage through the Lagrange points. Unfortunately, we have been unable to conclusively show how the phase angles become clumped since a reliable measure of $\theta_{R}$ along all points of the orbit is unavailable to us. That the clumping occurs during the formation of the bar is, however, beyond doubt. Since it is likely associated with bar-induced spirals, we suggest that clumping may be responsible for the incomplete, or partial, rings in barred galaxies (the "pseudorings" or $\mathrm{R}^{\prime}$ rings of Buta (1986)). It has been shown that these rings can be generated by stars ejected along the unstable manifolds (e.g., Athanassoula 2012). If the rate of ejection from the Lagrange points is not constant (due to, say, clumping in the phase angle), then we expect incomplete rings to form. The clumping maintains itself by counteracting phase mixing. The transient overdensities provide a force that alters the orbit's actions and corresponding frequencies. Since we have used very little prior information in extracting these resonant orbits (only the choice of normalisation), we have preserved the time dependent transient nature of processes in the disc.

The release of the Gaia (Perryman et al. 2001) and LAMOST (Deng 2014) datasets will herald a dramatic advance in our knowledge of the kinematic landscape, not only in the Solar vicinity but also to the very edges of the disc. For this reason, it is vital that we have at our disposal the tools required to analyse fully the output from increasingly sophisticated N-body simulations. Deviations from axisymmetry and the resulting resonances must be well understood if we are to comprehend how the Milky Way has evolved. Improving our knowledge of the kinematic structures will help us understand better the importance of secular mechanisms at work in the disc. Decomposing N-body discs into their major orbital components gives us the opportunity to begin to build up analytic models of complex systems by superposing many distinct distribution functions. The method presented here for dissecting simulations should serve as a complementary tool to more established techniques and has the potential to fill in the gaps in our knowledge of galaxy evolution that have yet to be illuminated.

We are deeply indebted to Marcel Zemp \& Zhao$\mathrm{Yu}$ Li for many helpful discussions and support during this project. We also thank the referee for insightful comments which greatly improved the paper. The authors acknowledge financial support from the CAS One Hundred Talent Fund and NSFC Grants 11173002, 11333003,11322326 and 11073037 . This work was also supported by the following grants: the Gaia Research for European Astronomy Training (GREAT-ITN) Marie Curie network, funded through the European Union Seventh Framework Programme (FP7/2007-2013) under grant agreement no 264895; the Strategic Priority Research Program "The Emergence of Cosmological Structures" of the Chinese Academy of Sciences, Grant No. XDB09000000; and the National Key Basic Research Program of China 2014CB845700. This work made use of the super-computing facilities at Shanghai Astronomical Observatory.

\section{REFERENCES}

Ahn, C. P., Alexandroff, R., Allende Prieto, C., et al. 2012, ApJS, 203, 21

Arnol'd, V. I. 1963, Russian Mathematical Surveys, 18, 85

Athanassoula, E. 2002, ApJ, 569, L83

—. 2003, MNRAS, 341, 1179

-. 2012, MNRAS, 426, L46

Athanassoula, E., Romero-Gómez, M., Bosma, A., \& Masdemont, J. J. 2009a, MNRAS, 400, 1706

Athanassoula, E., Romero-Gómez, M., \& Masdemont, J. J. 2009b, MNRAS, 394, 67 
Belokurov, V., Walker, M. G., Evans, N. W., et al. 2010, ApJ, 712, L103

Binney, J., \& Tremaine, S. 2008, Galactic Dynamics: Second Edition (Princeton University Press)

Bissantz, N., \& Gerhard, O. 2002, MNRAS, 330, 591

Blitz, L., \& Spergel, D. N. 1991, ApJ, 379, 631

Bond, N. A., Ivezić, Ž., Sesar, B., et al. 2010, ApJ, 716 , 1

Bovy, J., Bird, J. C., García Pérez, A. E., \& Zasowski, G. 2014a, ArXiv e-prints, arXiv:1410.8135

Bovy, J., Rix, H.-W., \& Hogg, D. W. 2012a, ApJ, 751, 131

Bovy, J., Rix, H.-W., Liu, C., et al. 2012b, ApJ, 753, 148 Bovy, J., Nidever, D. L., Rix, H.-W., et al. 2014b, ApJ, 790,127

Buta, R. 1986, ApJS, 61, 609

Cao, L., Mao, S., Nataf, D., Rattenbury, N. J., \& Gould, A. 2013, MNRAS, 434, 595

Carollo, D., Beers, T. C., Chiba, M., et al. 2010, ApJ, 712,692

Ceverino, D., \& Klypin, A. 2007, MNRAS, 379, 1155

Chirikov, B. V. 1979, Phys. Rep., 52, 263

Collett, J. L., Dutta, S. N., \& Evans, N. W. 1997, MN-

RAS, 285, 49

Contopoulos, G. 1970, ApJ, 160, 113

—. 1980, A\&A, 81, 198

de Jong, J. T. A., Yanny, B., Rix, H.-W., et al. 2010, ApJ, 714, 663

Debattista, V. P., Mayer, L., Carollo, C. M., et al. 2006, ApJ, 645, 209

Dehnen, W. 1999, ApJ, 524, L35

-. 2000, AJ, 119, 800

Dehnen, W., \& Binney, J. J. 1998, MNRAS, 298, 387

Deng, L. 2014, in IAU Symposium, Vol. 298, IAU Symposium, ed. S. Feltzing, G. Zhao, N. A. Walton, \& P. Whitelock, 269-280

Dwek, E., Arendt, R. G., Hauser, M. G., et al. 1995, ApJ, 445, 716

Eisenstein, D. J., Weinberg, D. H., Agol, E., et al. 2011, AJ, 142,72

Englmaier, P., \& Gerhard, O. 1999, MNRAS, 304, 512

Eskridge, P. B., Frogel, J. A., Pogge, R. W., et al. 2000, A.J, 119,536

Evans, N. W., \& Collett, J. L. 1993, MNRAS, 264, 353

Freudenreich, H. T. 1998, ApJ, 492, 495

Gardner, E., Debattista, V. P., Robin, A. C., Vásquez, S., \& Zoccali, M. 2014, MNRAS, 438, 3275

Gerhard, O., \& Martinez-Valpuesta, I. 2012, ApJ, 744, L8

Gerhard, O. E., \& Saha, P. 1991, MNRAS, 251, 449

Gómez, G., Koon, W. S., Lo, M. W., et al. 2004, Nonlinearity, 17, 1571

Hohl, F. 1971, ApJ, 168, 343

Howard, C. D., Rich, R. M., Reitzel, D. B., et al. 2008, ApJ, 688, 1060

Howes, L. M., Asplund, M., Casey, A. R., et al. 2014, MNRAS, 445, 4241

Kalnajs, A. J. 1978, in IAU Symposium, Vol. 77, Structure and Properties of Nearby Galaxies, ed. E. M. Berkhuijsen \& R. Wielebinski, 113-125

Kawata, D., Hunt, J. A. S., Grand, R. J. J., Pasetto, S., \& Cropper, M. 2014, MNRAS, 443, 2757

Koposov, S. E., Rix, H.-W., \& Hogg, D. W. 2010, ApJ, 712,260

Li, Z.-Y., \& Shen, J. 2012, ApJ, 757, L7

Li, Z.-Y., Shen, J., Rich, R. M., Kunder, A., \& Mao, S. 2014, ApJ, 785, L17
Lichtenberg, A. J., \& Lieberman, M. A. 1983, Regular and stochastic motion

Liu, C., Xue, X., Fang, M., et al. 2012, ApJ, 753, L24

Lopez-Corredoira, M., Abedi, H., Garzon, F., \& Figueras, F. 2014, ArXiv e-prints, arXiv:1409.6222

Lynden-Bell, D. 1973, in Saas-Fee Advanced Course 3: Dynamical Structure and Evolution of Stellar Systems, ed. G. Contopoulos, M. Henon, \& D. Lynden-Bell, 91

Lynden-Bell, D., \& Kalnajs, A. J. 1972, MNRAS, 157, 1 Majewski, S. R., \& SDSS-III/APOGEE Collaboration. 2014, in American Astronomical Society Meeting Abstracts, Vol. 223, American Astronomical Society Meeting Abstracts, 440.01

Marinova, I., \& Jogee, S. 2007, ApJ, 659, 1176

Martinez-Valpuesta, I., Shlosman, I., \& Heller, C. 2006, ApJ, 637, 214

Minchev, I., Boily, C., Siebert, A., \& Bienayme, O. 2010, MNRAS, 407, 2122

Minchev, I., Famaey, B., Quillen, A. C., et al. 2012, A\&A, 548, A126

Molloy, M., Smith, M., Shen, J., \& Evans, W. in prep

Monari, G., Helmi, A., Antoja, T., \& Steinmetz, M. 2014, ArXiv e-prints, arXiv:1402.4479

Newberg, H. J., Yanny, B., \& Willett, B. A. 2009, ApJ, 700, L61

Nidever, D. L., Zasowski, G., Majewski, S. R., et al. 2012, ApJ, 755, L25

Nidever, D. L., Bovy, J., Bird, J. C., et al. 2014, ApJ, 796, 38

Palmer, P. L. 1994, in Lecture Notes in Physics, Berlin Springer Verlag, Vol. 433, Galactic Dynamics and NBody Simulations, ed. G. Contopoulos, N. K. Spyrou, \& L. Vlahos, 143-189

Perryman, M. A. C., de Boer, K. S., Gilmore, G., et al. 2001, A\&A, 369, 339

Quillen, A. C., Dougherty, J., Bagley, M. B., Minchev, I., \& Comparetta, J. 2011, Monthly Notices of the Royal Astronomical Society, 417, 762

Raha, N., Sellwood, J. A., James, R. A., \& Kahn, F. D. 1991, Nature, 352, 411

Randich, S., Gilmore, G., \& Gaia-ESO Consortium. 2013, The Messenger, 154, 47

Rich, R. M., Reitzel, D. B., Howard, C. D., \& Zhao, H. 2007, ApJ, 658, L29

Robin, A. C., Marshall, D. J., Schultheis, M., \& Reylé, C. 2012, A\&A, 538, A106

Rojas-Arriagada, A., Recio-Blanco, A., Hill, V., et al. 2014, A\&A, 569, A103

Romero-Gómez, M., Athanassoula, E., Masdemont, J. J., \& García-Gómez, C. 2007, A\&A, 472, 63

Romero-Gómez, M., Masdemont, J. J., Athanassoula, E., \& García-Gómez, C. 2006, A\&A, 453, 39

Saito, R. K., Zoccali, M., McWilliam, A., et al. 2011, AJ, 142,76

Sellwood, J. A. 1981, A\&A, 99, 362

Sellwood, J. A., \& Wilkinson, A. 1993, Reports on Progress in Physics, 56, 173

Shen, J., Rich, R. M., Kormendy, J., et al. 2010, ApJ, 720, L72

Siebert, A., Famaey, B., Minchev, I., et al. 2011, MNRAS, 412, 2026

Smith, M. C., Whiteoak, S. H., \& Evans, N. W. 2012, ApJ, 746, 181

Smith, M. C., Evans, N. W., Belokurov, V., et al. 2009, MNRAS, 399, 1223

Stanek, K. Z., Udalski, A., Szymanski, M., et al. 1997, ApJ, 477, 163 
Steinmetz, M., Zwitter, T., Siebert, A., et al. 2006, AJ, 132,1645

Tagger, M., Sygnet, J. F., Athanassoula, E., \& Pellat, R. 1987, ApJ, 318, L43

Toomre, A. 1981, in Structure and Evolution of Normal Galaxies, ed. S. M. Fall \& D. Lynden-Bell, 111-136

Vásquez, S., Zoccali, M., Hill, V., et al. 2013, A\&A, 555, A91

Wang, Y., Mao, S., Long, R. J., \& Shen, J. 2013, MN-
RAS, 435, 3437

Weinberg, M. D. 1994, ApJ, 420, 597

Weiner, B. J., \& Sellwood, J. A. 1999, ApJ, 524, 112

Yanny, B., Rockosi, C., Newberg, H. J., et al. 2009a, AJ, 137,4377

Yanny, B., Newberg, H. J., Johnson, J. A., et al. 2009b, ApJ, 700, 1282 